ARTICLE

\title{
Human aminolevulinate synthase structure reveals a eukaryotic-specific autoinhibitory loop regulating substrate binding and product release
}

Henry J. Bailey 1,5, Gustavo A. Bezerra1,5, Jason R. Marcero2,5, Siladitya Padhi (0) 3,5, William R. Foster (1) ${ }^{1}$, Elzbieta Rembeza1, Arijit Roy (1) ${ }^{3}$, David F. Bishop ${ }^{4}$, Robert J. Desnick ${ }^{4}$, Gopalakrishnan Bulusu (1) ${ }^{3}$, Harry A. Dailey $\mathrm{Jr}^{2} \&$ Wyatt W. Yue (i) ${ }^{1 凶}$

$5^{\prime}$-aminolevulinate synthase (ALAS) catalyzes the first step in heme biosynthesis, generating 5'-aminolevulinate from glycine and succinyl-CoA. Inherited frameshift indel mutations of human erythroid-specific isozyme ALAS2, within a C-terminal (Ct) extension of its catalytic core that is only present in higher eukaryotes, lead to gain-of-function $X$-linked protoporphyria (XLP). Here, we report the human ALAS2 crystal structure, revealing that its Ctextension folds onto the catalytic core, sits atop the active site, and precludes binding of substrate succinyl-CoA. The Ct-extension is therefore an autoinhibitory element that must re-orient during catalysis, as supported by molecular dynamics simulations. Our data explain how $\mathrm{Ct}$ deletions in XLP alleviate autoinhibition and increase enzyme activity. Crystallography-based fragment screening reveals a binding hotspot around the Ct-extension, where fragments interfere with the Ct conformational dynamics and inhibit ALAS2 activity. These fragments represent a starting point to develop ALAS2 inhibitors as substrate reduction therapy for porphyria disorders that accumulate toxic heme intermediates.

\footnotetext{
${ }^{1}$ Structural Genomics Consortium, Nuffield Department of Medicine, University of Oxford, Oxford OX3 7DQ, UK. ${ }^{2}$ Department of Biochemistry and Molecular Biology, University of Georgia, Athens, GA 30602, USA. ${ }^{3}$ TCS Innovation Labs-Hyderabad (Life Sciences Division), Tata Consultancy Services Ltd, Hyderabad 500081, India. ${ }^{4}$ Department of Genetics and Genomics Sciences, Icahn School of Medicine at Mount Sinai, New York, NY 10029, USA. ${ }^{5}$ These

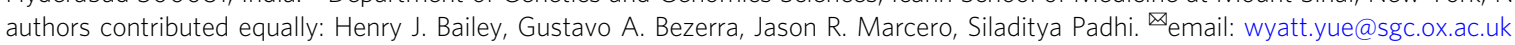


T he tetrapyrrole cofactor heme is essential for various cellular processes across lifeforms. In metazoans, heme synthesis occurs via a conserved eight-reaction pathway that requires iron, glycine, and succinyl-CoA. The first and ratelimiting step is carried out by $5^{\prime}$-aminolevulinate synthase (ALAS; EC 2.3.1.37) in the mitochondria ${ }^{1,2}$. ALAS catalyses the pyridoxal $5^{\prime}$-phosphate (PLP)-dependent condensation of succinyl-CoA and glycine to form aminolevulinic acid (ALA), with CoA and $\mathrm{CO}_{2}$ as by-products. The product ALA is then transported into the cytoplasm for the subsequent biosynthetic steps that eventually lead back into the mitochondria, where ferrochelatase (FECH) ultimately inserts iron into protoporphyrin IX (PPIX) to produce heme $e^{3,4}$. Vertebrates have evolved two nuclear-encoded ALAS isozymes 5,6 that share $\sim 60 \%$ amino acid sequence identity. ALAS1 (gene location 3p21.2) is the housekeeping enzyme providing a basal level of heme in non-erythroid cell types for cytochromes and other hemoproteins. ALAS2 (gene location $\mathrm{Xp11.21)}$ is predominantly expressed in erythroid progenitor cells, and synthesizes $85-90 \%$ of total body heme specifically for hemoglobin production during erythropoiesis. Mutations in the heme biosynthetic genes, resulting in an accumulation of toxic porphyrin intermediates, are associated with a group of inherited disorders called porphyrias ${ }^{3,7}$.

The ALAS catalytic mechanism, involving Schiff-base formation between the aldehyde carbonyl of the PLP cofactor and $\varepsilon$-amino group of a conserved lysine residue, has been well characterized $^{8-11}$. Without substrates, PLP is covalently attached to the active site lysine (Lys391 in human ALAS2 (hsALAS2)) as an internal aldimine adduct. The reaction begins with the glycine substrate replacing lysine in the Schiff-base linkage to form an external aldimine. Next, deprotonation of glycine forms a transient quinonoid intermediate that is competent for nucleophilic attack on the co-substrate, succinyl-CoA, resulting in a condensation reaction releasing the CoA moiety, followed by a decarboxylation assisted by the active site histidine (His285 in hsALAS2). Subsequent protonation regenerates the internal aldimine, and allows release of the product, ALA. A number of kinetic studies have suggested that the ALAS rate-determining step is product (ALA) release ${ }^{8,9}$. The structure of $R$. capsulatus ALAS (rcALAS) was the first reported for the enzyme ${ }^{12}$, revealing an induced-fit mechanism upon substrate binding, via an opento-close transition of a mobile active site loop. Conformational mobility of this loop has also been proposed to be a kinetic barrier for product release $8,11,13$.

Eukaryotic ALAS enzymes have evolved extensions appending to both $\mathrm{N}$ - and C-termini of the highly conserved catalytic core $\mathrm{e}^{14}$ (Fig. 1a). The N-terminal extensions, harboring the mitochondrial targeting sequence ${ }^{14-17}$, are poorly conserved between higher and lower eukaryotic ALAS enzymes, as well as between metazoan ALAS1 and ALAS2 (Supplementary Fig. 1). Metazoan enzymes further encode three Cys-Pro motifs ${ }^{18}$, two of which are within the targeting sequence. The third Cys-Pro motif, together with an invariant Gln-Glu-Asp-Val motif, are found within a $70-90$ aa region of poor sequence conservation and unknown function. In both ALAS1 and ALAS2, the Cys-Pro motifs have been shown to be responsible for heme-dependent inhibition of mitochondrial translocation of the enzyme precursor ${ }^{19,20}$.

The eukaryotic extension at the C-terminus (Ct-extension) ranges from 35 to 60 aa in length. Metazoan ALAS1 and ALAS2 share $\sim 53 \%$ sequence identity in their Ct-extensions (their major differences are found in the last 9 amino acids), while $S$. cerevisiae encodes an entirely different Ct-extension from metazoans, and also from S. pombe (Supplementary Fig. 1). Frameshift indel mutations in exon 11 of the human ALAS2 gene (on chromosome Xp11.21) that result in deletion, replacement, or elongation of its Ctextension are the molecular cause of X-linked protoporphyria (XLP,
MIM 300752), an inherited disorder that presents with painful phototoxicity and an increased risk for liver dysfunction and failure $^{21,22}$, due to high levels of the toxic heme intermediate PPIX. At the protein level, these genetic lesions cause enhanced enzyme activity, lending to XLP being referred to as a gain-of-function (GOF) disorder ${ }^{23-25}$. Recently, a GOF XLP phenotype was reported for mutations of the mitochondrial ATP-dependent Clp protease ClpX, an AAA + (ATPases associated with diverse cellular activities) unfoldase shown to activate ALAS dimers through partial unfolding of the enzyme to enhance loading of the PLP cofactor into the active site $^{26,27}$. XLP contrasts with another ALAS2-associated blood disorder, X-linked sideroblastic anemia (XLSA, MIM 300751), which results in loss of enzyme function and is characterized by heme-deficient and iron-overloaded red blood cells (ringed sideroblasts ${ }^{28-31}$. XLSA is attributable to mutations within exons 5-11 (including a few in the Ct-extension; from Human Gene Mutation Database) $^{32}$ that are predominantly missense in nature.

While the dichotomous genotype-phenotype correlation of XLSA and XLP is now widely recognized, it remains unknown how the GOF phenotypes in XLP are brought about by various mutations in the ALAS2 Ct-extension, although a self-inhibitory role has been proposed for this region ${ }^{33}$. The recently published ALAS2 structure from S. cerevisiae (scALAS) showed each Ctextension wrapping around the surface of the ALAS homodimer, albeit distant from its active site ${ }^{34}$. This sequence, furthermore, bears no homology with other eukaryotic counterparts and is likely to be evolutionarily distinct from the mammalian ALAS2 Ct-extension. Importantly, deletion of the scALAS extension does not increase its enzyme activity ${ }^{34}$, contrary to the XLP-causing mutations in hsALAS2. Here, we determine the crystal structure of hsALAS2 which, in combination with molecular dynamics simulations and enzyme kinetics, reveals salient features of the Ct-extension that explain its role in enzyme regulation and in causing XLP.

\section{Results}

hsALAS2 without presequence is sufficient for catalysis. We expressed in E. coli, as soluble proteins, several constructs of hsALAS2 with different truncations within the $\mathrm{N}$-terminal extension, while keeping their Ct-extensions intact (Supplementary Fig. 2). The isolated as-purified proteins showed varying degrees of proteolysis (see below), as observed previously ${ }^{24}$, which we attributed to cleavage of the 40 -aa Ct-extension. For crystallography experiments, we next employed baculovirus-infected insect Sf9 cells to express hsALAS2 aa 79-587 (hsALAS2 ${ }_{\triangle \mathrm{N} 78}$ ) and aa 143-587 (hsALAS2 ${ }_{\triangle \mathrm{N} 142}$ ) resulting in soluble proteins with no signs of proteolytic degradation. The majority of the as-purified hsALAS2 $2_{\triangle N 78}$ and hsALAS2 ${ }_{\triangle N 142}$ proteins from insect cells contain the PLP cofactor, with their Schiff base predominantly in the deprotonated enolimine tautomer ${ }^{35}$ (Supplementary Fig. 3). Insect cell-expressed hsALAS2 is active with $K_{\mathrm{m}}$ values $(31 \pm 4 \mu \mathrm{M}$ for succinyl-CoA, $11.8 \pm 1.0 \mu \mathrm{M}$ for glycine) close to those reported by others using a similar assay ${ }^{24}$ (Supplementary Fig. 4a, b). This form of the enzyme is also capable of forming an interaction with the previously reported partner, beta subunit (SUCLA2) of the succinyl-CoA synthetase complex SUCLG1-SUCLA2 (refs. ${ }^{36,37}$ ), as revealed by cross-linking using disuccinimidyl glutarate (Supplementary Fig. 4c).

Overall structure of hsALAS2 and conserved features. We first crystallized the hsALAS2 ${ }_{\triangle \mathrm{N} 78}$ protein and determined its crystal structure at $2.7 \AA$ resolution by molecular replacement, using the rcALAS structure as a search model. Unexpectedly, the N-terminal residues $79-142$ of our construct were not visible in the electron density. Intact mass spectrometry of purified hsALAS2 ${ }_{\triangle \mathrm{N} 78}$ 


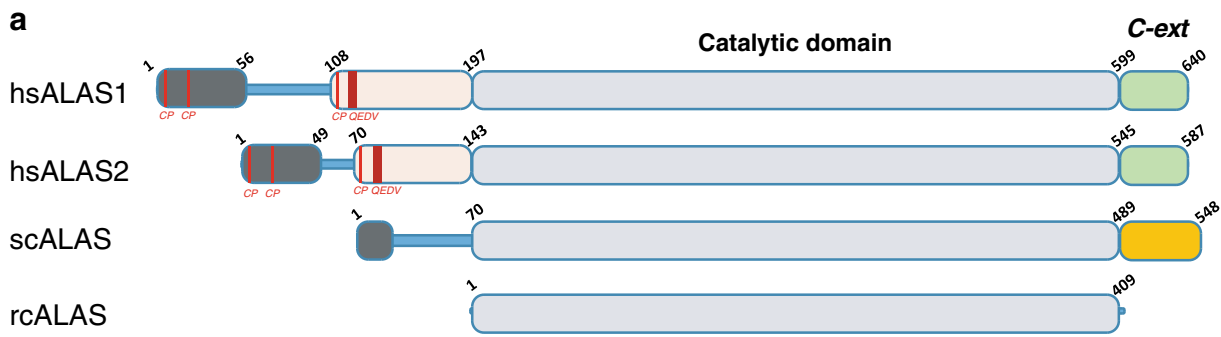

b

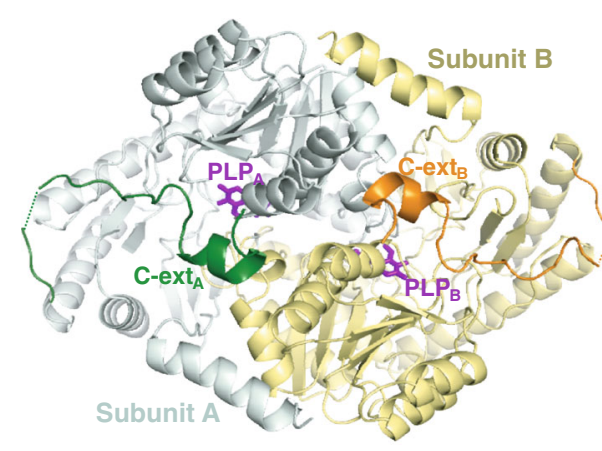

d

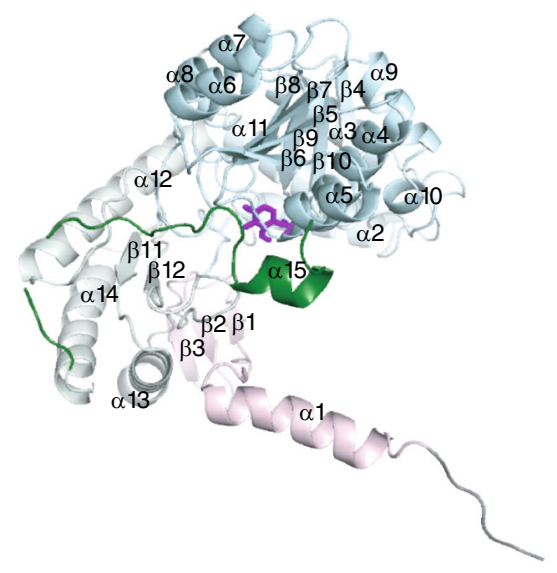

c

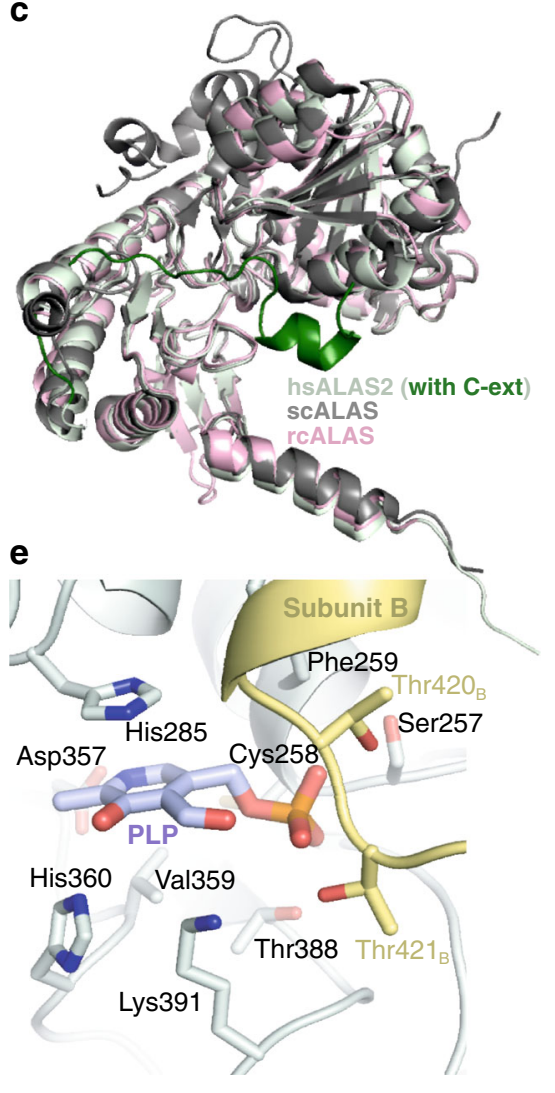

Fig. 1 Domain organization and structure of hsALAS2. a Domain architecture of hsALAS1, hsALAS2, scALAS, and rcALAS, highlighting the catalytic core (gray box) flanked by N-terminal (black, pink boxes) and C-terminal extension (green in higher eukaryotes, orange in lower eukaryotes). b hsALAS2 homodimer (this study) composed of monomer A (catalytic domain in gray, Ct-extension in dark green) and the opposite monomer B (catalytic domain in yellow, Ct-extension in orange. PLP is shown in purple sticks. c Structure superimposition of protomer from hsALAS2 (this study), scALAS (PDB 5TXR) and rCALAS (PDB 2BWN). d Domain organization and secondary structure assignment for hsALAS2. Subdomain 1 is shown in pink, subdomain 2 in gray, subdomain 3 in cyan, and the Ct-extension in dark green. e PLP binding site of hsALAS2. PLP-interacting residues from monomer A are shown in gray and from opposing monomer B in yellow. PLP is shown in mauve (carbon color).

nonetheless indicates that aa $79-142$ are present (Supplementary Fig. 5a), suggesting this region remains intact in the crystal, albeit disordered. This is further revealed by crystal packing analysis of ALAS2 protomers in the crystal lattice, leaving space for the Nterminal region (Supplementary Fig. 5b). Subsequently, the shorter hsALAS2 ${ }_{\triangle \mathrm{N} 142}$ protein was crystallized, and its higher resolution structure at $2.3 \AA$ resolution was determined (Table 1) and will be referred to hereafter in the manuscript.

The crystal asymmetric unit contains an hsALAS2 homodimer (Fig. 1b), as shown in solution by size exclusion chromatography and small angle X-ray scattering (SAXS)(Supplementary Fig. 6, Supplementary Table 1). The two monomers (rmsd $0.167 \AA$ ) are tightly interlocked in the structure, burying a total of $4850 \AA^{2}$ accessible surface per monomer. The dimer interface is largely derived from the catalytic domain and highly conserved with the rcALAS structure (Supplementary Fig. 1), although the
Ct-extension contributes three aromatic residues that mediate inter-monomer interactions (see next section). This said, an hsALAS2 construct encoding the catalytic domain alone (aa $143-545$, hsALAS2 $\left.{ }_{\triangle \mathrm{N} 142 \Delta \mathrm{C} 545}\right)$ is sufficient to homodimerize in solution (Supplementary Fig. 6b, e).

The catalytic core of hsALAS2 is highly similar to rcALAS (sequence identity $50 \%$, rmsd $1.1 \AA$ ) and to scALAS $(45 \%, 1.5 \AA$ ) (Fig. 1c). Together they are part of the superfamily of type I PLPdependent enzymes, in which the catalytic core can be dissected into three sub-domains. In hsALAS2 (Fig. 1d), they correspond to: subdomain 1 (aa 143-195) forming an $\alpha$-helix and a three-stranded, antiparallel $\beta$-sheet; the large central subdomain 2 (aa 196-439) forming a seven-stranded $\beta$-sheet enclosed by nine $\alpha$-helices in repeated $\beta / \alpha$ motifs; and the last subdomain 3 (aa 440-544) including a three-stranded antiparallel $\beta$-sheet, three $\alpha$-helices and a fourth $\beta$-strand that contributes to the sub-domain $1 \beta$-sheet. 


\section{Table 1 Data collection and refinement statistics for} hsALAS2 $_{\Delta \text { N142 }}$ and hsALAS2 ${ }_{\Delta \text { N78 }}$ crystal structures.

\begin{tabular}{|c|c|c|}
\hline Dataset & hsALAS2 $_{\Delta \text { N142 }}$ & hsALAS2 $_{\Delta \text { N78 }}$ \\
\hline Beamline & $\begin{array}{l}\text { Diamond } \\
\text { beamline } 104\end{array}$ & $\begin{array}{l}\text { Diamond } \\
\text { beamline } 124\end{array}$ \\
\hline Wavelength $(\AA)$ & 0.9795 & 0.9795 \\
\hline $\begin{array}{l}\text { Unit cell parameters } \\
(a, b, c)\end{array}$ & $\begin{array}{l}125.77 \AA, 107.7 \AA, \\
75.71 \AA\end{array}$ & $\begin{array}{l}78.21 \AA, 155.62 \AA, \\
86.24 \AA\end{array}$ \\
\hline$(\alpha, \beta, \gamma)$ & $90.0^{\circ}, 109.0^{\circ}, 90.0^{\circ}$ & $\begin{array}{l}90.0^{\circ}, \\
98.45^{\circ}, 90.0^{\circ}\end{array}$ \\
\hline Space group & C 121 & P 1211 \\
\hline Resolution range $(\AA)$ & $71.53-2.30$ & $85.32-2.65^{a}$ \\
\hline Unique reflections & 40,141 & 58,818 \\
\hline Rsym (\%) & $0.09(0.71)$ & $0.21(0.76)$ \\
\hline $\mathrm{I} / \operatorname{sig}(\mathrm{I})$ & $5.8(1.0)$ & $4.9(1.5)$ \\
\hline Completeness (\%) & $94.8(95.0)$ & $72.6(19.3)^{a}$ \\
\hline Multiplicity & $2.3(2.3)$ & $3.4(3.0)$ \\
\hline$R_{\text {cryst }}(\%)$ & 0.21 & 0.21 \\
\hline$R_{\text {free }}(\%)$ & 0.23 & 0.26 \\
\hline Wilson $B$ factor $\left(\AA^{2}\right)$ & 43.16 & 26.8 \\
\hline $\begin{array}{l}\text { Average total } B \text { factor } \\
\left(\AA^{2}\right)\end{array}$ & 55.2 & 25.8 \\
\hline R.m.s.d. bond length $\left(\AA^{2}\right)$ & 0.003 & 0.004 \\
\hline R.m.s.d. bond angle $\left({ }^{\circ}\right)$ & 0.69 & 0.68 \\
\hline Clashscore & 6.7 & 5.78 \\
\hline $\begin{array}{l}\text { Ramachadran } \\
\text { favored (\%) }\end{array}$ & 95.6 & 95.6 \\
\hline $\begin{array}{l}\text { Ramachandran } \\
\text { disallowed (\%) }\end{array}$ & 0 & 0 \\
\hline Rotamer outliers (\%) & 0 & 0.15 \\
\hline
\end{tabular}

Data from highest resolution shell shown in parenthesis.

anisotropic data truncated in STARANISO using local I/sigl cut off at 1.2 results in the inclusion of data to 2.65 with low isotropic completeness.

Like its orthologs, hsALAS2 is an obligate homodimer in that each active site is formed from both monomeric subunits, with one PLP molecule bound per active site. Prior to crystallization, the protein was treated with hydroxylamine to convert the covalently-bound PLP (in equilibrium between two tautomers; Supplementary Fig. 3) into the non-covalent form that is more homogenous (Supplementary Fig. 7). As expected from the sequence alignment (Supplementary Fig. 1), hsALAS2 employs a highly conserved set of interactions with PLP, involving mainly the central sub-domain of the catalytic core (Fig. 1e). In short, the pyridinium ring fits snugly through hydrophobic interactions with His285 and Val359, as well as hydrogen bonds with His360 and Asp357. The PLP phosphate is neutralized by four hydrogen bonds from its own subunit (Ser257 and Thr388 side-chains, Cys258 and Phe259 main-chains), as well as three hydrogen bonds contributed from the opposing monomer (Thr420 and Thr421 side-chains, Thr421 main-chain).

hsALAS2 C-terminus is a self-inhibitory loop. The largest structural difference between hsALAS2 and homologs resides in the C-terminus following the catalytic core. The 40-residue hsALAS2 Ct-extension (Fig. 1b and Supplementary Fig. 8) begins with a poorly structured segment (aa 544-557) which stretches outward to the protein exterior. There is no clear electron density for part of this segment (aa 549-555, Supplementary Fig. 8a), suggesting its mobility. The next segment of the Ct-extension (aa 560-568, Supplementary Fig. 8b) threads across the interface of sub-domains 2 and 3 as a linear polypeptide, with several mainchain amides and carbonyls shielded by hydrogen-bonding with catalytic core residues (Arg479 and Glu513, which are not present in scALAS or rcALAS). A two-turn helix (a15; Ser568-Phe575) then ensues, forming a lid atop the PLP-bound active site, before being directed to the protein exterior (Phe575-Met578). Close examination of helix a15 (Fig. 2a) reveals that its two Glu residues, Glu569 and Glu571, form charged interactions respectively with Arg511 from subdomain 3 and Lys299/Arg293 from subdomain 2. This helix also contributes three aromatic residues Trp570, Tyr574, and Phe575 which stack among themselves through $\pi-\pi$ interactions, and anchor the dimerization interface through hydrophobic interactions with Phe267, Thr268, and Ala415 of the opposite dimer subunit. Additional hydrogen bonds were contributed from Phe575 and Gly576 (main chain), with Lys271 on the opposing subunit and Arg293, respectively. The last 9 residues of the hsALAS2 Ct-extension (aa 579-587) were not modeled in the structure, likely due to high disorder as they are surface-exposed. These residues also represent one of the largest sequence divergences between ALAS1 and ALAS2 (Supplementary Fig. 1).

The succinyl-CoA-bound rcALAS structure (PDB 2BWO $)^{12}$ revealed residues involved in substrate succinyl-CoA binding, which are nearly invariant across the phyla (based on sequence alignment in Supplementary Fig. 1), suggesting that hsALAS2 has evolved the same binding site. However, when superimposing hsALAS2 and succinyl-CoA-bound rcALAS structures, we observed to our surprise that the hsALAS2 succinyl-CoA site is blocked by helix $\alpha 15$ and the C-terminus (Fig. 2b), which would sterically overlap with the ADP-diphosphate moiety of a modeled succinyl-CoA molecule. Our hsALAS2 structure therefore represents a conformation of the Ct-extension that precludes binding of succinyl-CoA to the active site. The steric blockade is mediated by several interactions between helix $\alpha 15$ and the catalytic core (Fig. 2a). These include (i) salt bridges of Glu571 with both Lys299 and Arg293, blocking the expected position of the succinyl-CoA ADP adenine group, (ii) the hydrogen bond between Gly576 (main-chain) and Arg293 that blocks the $3^{\prime}$-phosphoadenosine moiety, and (iii) the salt bridge between Glu569 and Arg511. It is of note that Arg511, a residue from the mobile active site loop (aa 505-514), also forms a salt bridge with Asp159 from helix a1. In rcALAS, the equivalent Arg511:Asp159 interaction plays a role in succinyl-CoA-induced conformational change during catalysis ${ }^{12}$ (cf. Figure $3 a$ ).

The observed direct interaction between the Ct-extension and the active site loop in our hsALAS2 structure, through Arg511, implies that the mobility of the active site loop is driven by a conformational change of the Ct-extension, and suggests that these two regions act in concert to drive the ALAS2 catalytic cycle of substrate binding and product release. We explored further the role of R511 by site-directed mutagenesis on the crystallized construct, hsALAS2 $2_{\triangle \mathrm{N} 142}$, to generate the R511E variant which abolishes charged interactions with Glu569 (Ct-extension) and Asp159 (active site loop). Compared to wild-type (WT), the R511E variant is more prone to proteolysis at the Ct-extension as shown by SDSPAGE (Fig. 3b) and intact mass spectrometry (Supplementary Fig. $5 c, d$ ), suggesting that the interaction of the Ct-extesion with the catalytic domain active site loop has been significantly disrupted. Consistent with this, the $\mathrm{R} 511 \mathrm{E}$ variant is $>2{ }^{\circ} \mathrm{C}$ more thermolabile than WT protein, with melting temperature similar to the hsALAS2 ${ }_{\triangle \mathrm{N} 142 \Delta \mathrm{C} 545}$ (WT or R511E) protein with Ct-extension omitted from the construct (Fig. 3c). Furthermore, we showed by steady-state kinetics that the proteolysis-prone R511E variant has 2 -fold higher enzyme activity than WT, as indicated by $V_{\max }$ and $k_{\text {cat }}$ values (Fig. $3 \mathrm{~d}$, e and Table 2). Of additional note, the R511E variant demonstrates a lower specificity $\left(k_{\mathrm{cat}} / K_{\mathrm{m}}\right)$ for glycine than WT protein, while the opposite was observed for succinyl-CoA (Table 2). Altogether, our data suggest that the salt bridge network of Glu569:Arg511:Asp159 plays an important role in regulating the Ct-extension during catalysis. 
a

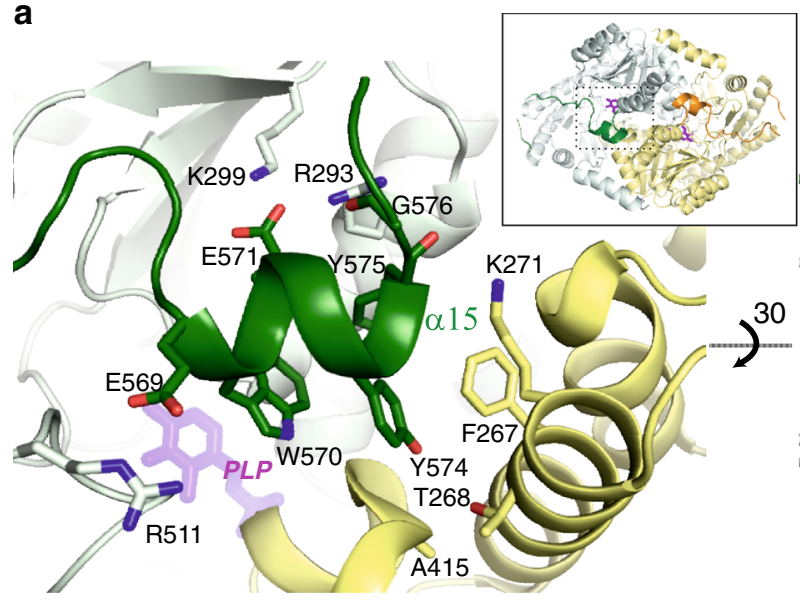

b
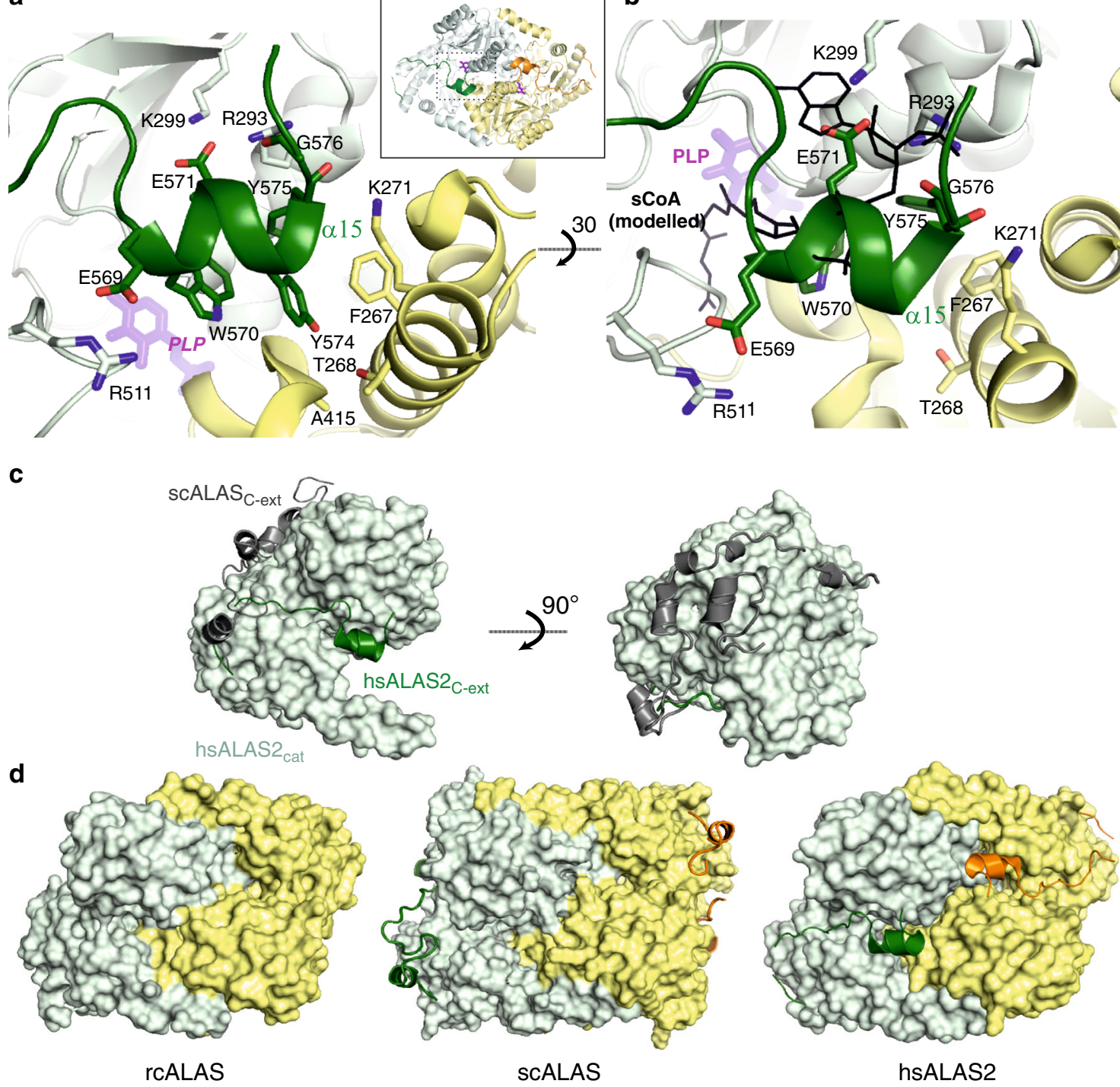

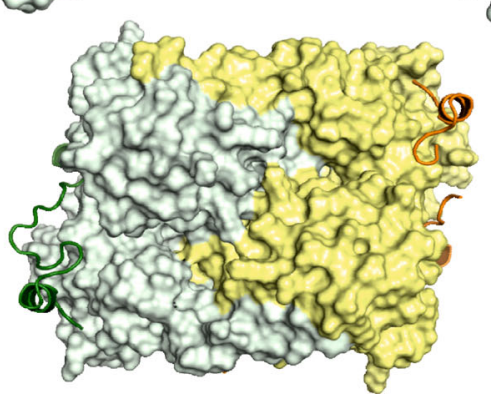

SCALAS

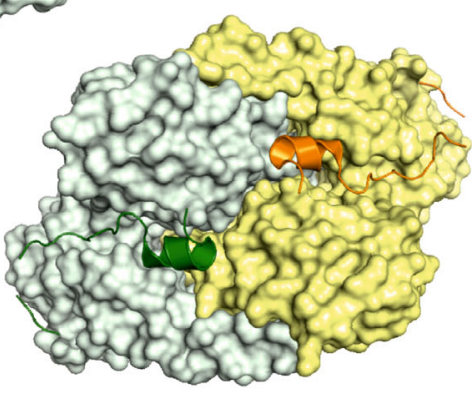

hsALAS2

Fig. 2 hsALAS2 C-terminal extension is mobile and interferes directly with active site. a Interactions of helix $\alpha 15$ from the Ct-extension (dark green) with the catalytic core of monomer A shown in light green and the opposing monomer B shown in yellow. $\mathbf{b}$ Similar view as panel $\mathrm{A}$, related by $30^{\circ}$ rotation along the horizontal axis. The succinyl-CoA ( $\mathrm{s} C \mathrm{~A}$ ) ligand, modeled into hsALAS2 from rCALAS (PDB 2BWO), is shown in black lines to highlight steric clashes with helix $\alpha 15$. $\mathbf{c}$ hsALAS2 monomer with catalytic domain in surface representation (light green) and Ct-extension in cartoon representation (dark green). The scALAS Ct-extension is also superimposed in cartoon representation (gray). d rcALAS (PDB 2BWN), scALAS (PDB 5TXR) and hsALAS2 (this study) homodimers. Catalytic domains are shown in surface representation (monomer A light green, monomer $B$ yellow). Ct-extensions are shown in cartoon representation for sCALAS and rCALAS (dark green for monomer A, orange for monomer B).

Comparison of our hsALAS2 structure with that of scALAS ${ }^{34}$ shows that their Ct-extensions not only differ in sequence (Supplementary Fig. 1) but also in structural locations and conformations (Fig. 2c, d). The point of structural divergence occurs after the last helix (a14) in the catalytic domain, at position Leu545 of hsALAS2 (Leu489 of scALAS). The hsALAS2 extension threads across the subdomain interface and shields the substrate binding site from the exterior (Fig. 2c, left). In scALAS, by contrast, a two-turn helix directs its Ct-extension back along the periphery of sub-domain 3, packs against a unique 22 -aa insertion in sub-domain 2 (aa 302-323, scALAS numbering) not present in hsALAS2 (Fig. 2c, right), and continues to wrap around the homodimer surface reaching as far as the N-terminal sub-domain 1 of the opposing monomer. Unlike hsALAS2, each Ct-extension in scALAS did not interact with its substrate binding site, leaving it exposed as in the rcALAS structure (Fig. 2d).
Simulations of the ALAS2 C-terminal dynamics. We next employed molecular dynamics (MD) simulations to understand the mobility of the hsALAS2 Ct-extension. Based on the experimental structure, we modeled hsALAS2 in the apo form, the holo form with PLP covalently-bound to Lys391, and the ternary complex with glycine-PLP and succinyl-CoA. In the substratefree apo and holo forms, the Glu569:Arg511 and Glu571:Arg293/ Lys299 salt bridges, serving to stabilize the Ct-extension over the active site, remain intact for most of the duration of the simulations (Fig. 4a, left and Supplementary Figs. 9-11). In order for succinyl-CoA to bind and orientate in the active site, we reason that the Ct-extension must change its conformation. Indeed, simulation of the substrate-bound ternary complex shows complete breakage of the salt bridges (Fig. 4a, right and Supplementary Figs. 9-11), resulting in an open conformation that is structurally poised to bind the substrate succinyl-CoA (Fig. 4b and Supplementary Movies 1 and 2). We also carried out MD 
a

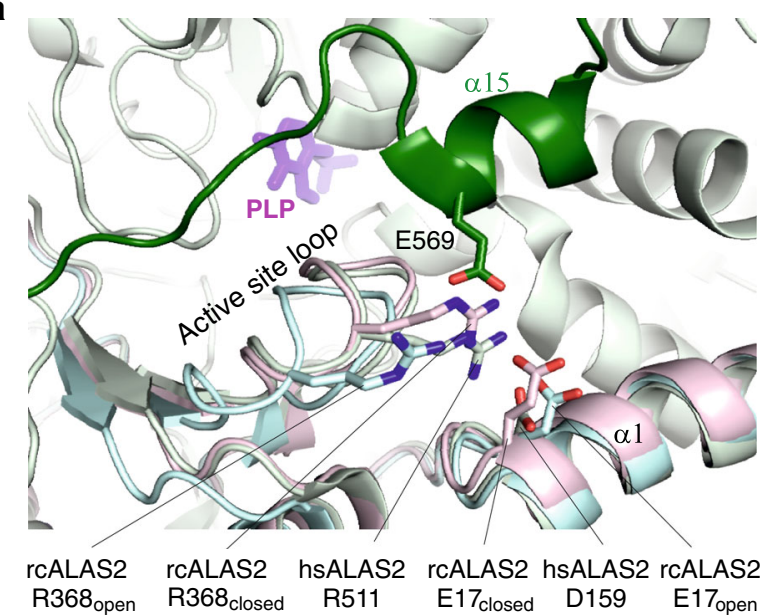

b $\quad$ hSALAS2 ${ }_{\triangle N 142}$

- hSALAS2 ${ }_{\triangle N 142 \triangle C 545}$

$\triangle$ hsALAS2 ${ }_{\triangle \mathrm{N} 142} \mathrm{R} 511 \mathrm{E}$

$\checkmark$ hsALAS2 ${ }_{\triangle N 142 \triangle C 545} R 511 E$
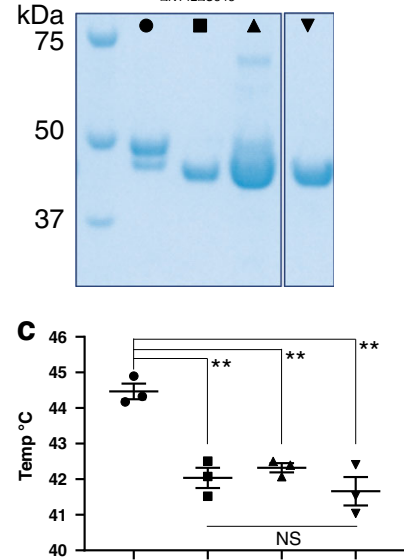
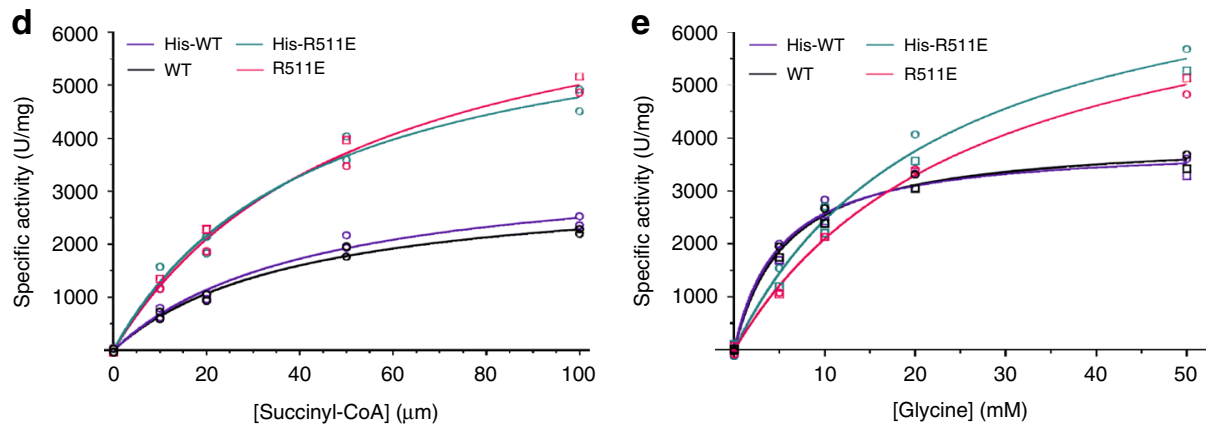

Fig. 3 Role of C-terminal extension in structural and functional integrity. a View of the hSALAS2 active site loop conformation within one monomer (catalytic domain light green, Ct-extension dark green). The active site loop from the open and closed forms of rcALAS (PDB 2BWN) are overlayed onto hsALAS2. b SDS-PAGE of the purified samples from hsALAS2 ${ }_{\triangle N 142}$ WT (square) and R511E variant (up triangle), as well as hsALAS2 $\triangle N 142 \Delta C 545$ WT (circle) and R511E variant (down triangle). c Midpoint melting temperatures, determined by differential scanning fluorimetry, of the four samples in panel b. Error bars represent mean values \pm SEM from $n=3$ technical replicates. ${ }^{\star *} p$-values determined by two sample $t$-test; $p=0.0026,0.0011$, and 0.0002 from left to right. d, e Progression curves for recombinant hsALAS2 ${ }_{\Delta N 142}$ WT and R511E variant, for both His-tagged and untagged proteins. Specific activity is equivalent to the initial velocity normalized to protein in each assay. Succinyl-CoA and glycine titrations contained $50 \mathrm{mM}$ glycine and $100 \mu \mathrm{M}$ succinylCoA, respectively. Each nonlinear regression line was fitted to data from two biological replicates $(n=2)$, with the data for each replicate (average of two technical replicates) plotted as distinct symbols (circle and square). Source data for Fig. 3b-e are provided as a Source Data file.

Table 2 Steady-state kinetics of recombinant hsALAS2 to study effect of His-tag and variants.

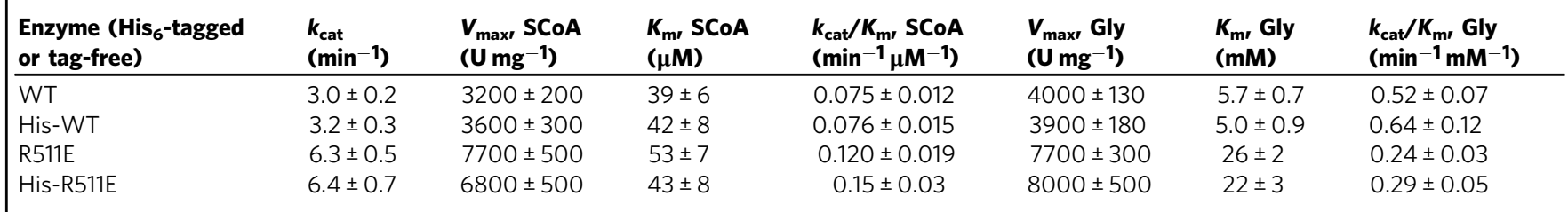

Kinetic parameters for recombinant N-terminal His 6 -tagged and tag-free hsALAS2 ${ }_{\Delta N 142}$ WT and R511E proteins. Reported parameters are best-fit values \pm SE for progression curves (Fig. $3 \mathrm{~d}_{\text {, }}$ e) derived from the Prism software (GraphPad 8.0). Source data are provided as a Source Data file.

simulations on a hsALAS2 R511E model in which the salt bridge with Glu569 would not be formed, to support our experimental observations that Arg511 is important for the integrity of Ctextension (see previous section and Fig. 3). Indeed, the accessibility of the Ct-extension in the R511E model is higher than WT, in that the R511E Ct-extension has moved away from the catalytic core over the course of the simulation, and this movement leads to an increase in the solvent accessible surface area (Supplementary Fig. 12).
During MD simulations, dimeric hsALAS2 exhibits asymmetry between the two subunits, a feature previously reported for murine ALAS2 (ref. ${ }^{38}$ ). This asymmetry is particularly observed in the dynamics of salt bridges from the two subunits of the ternary complex (Supplementary Figs. 10 and 11, compare bottom left and right panels). Asymmetry is also observed at the secondary structure level. Analysis of dynamic cross correlation maps (DCCM), which quantify correlations in motions between different regions of a protein $^{39}$, shows that covalent attachment of PLP to the active site 
a

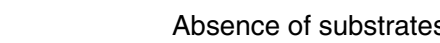

Presence of substrates

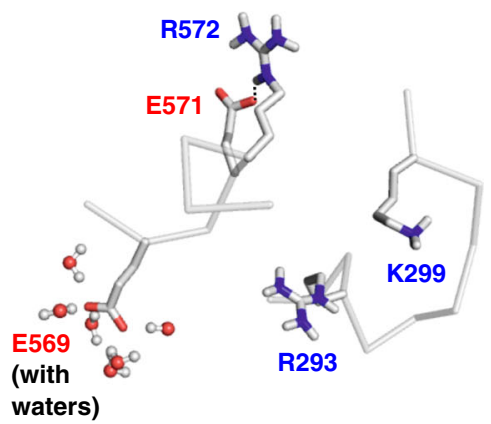

b

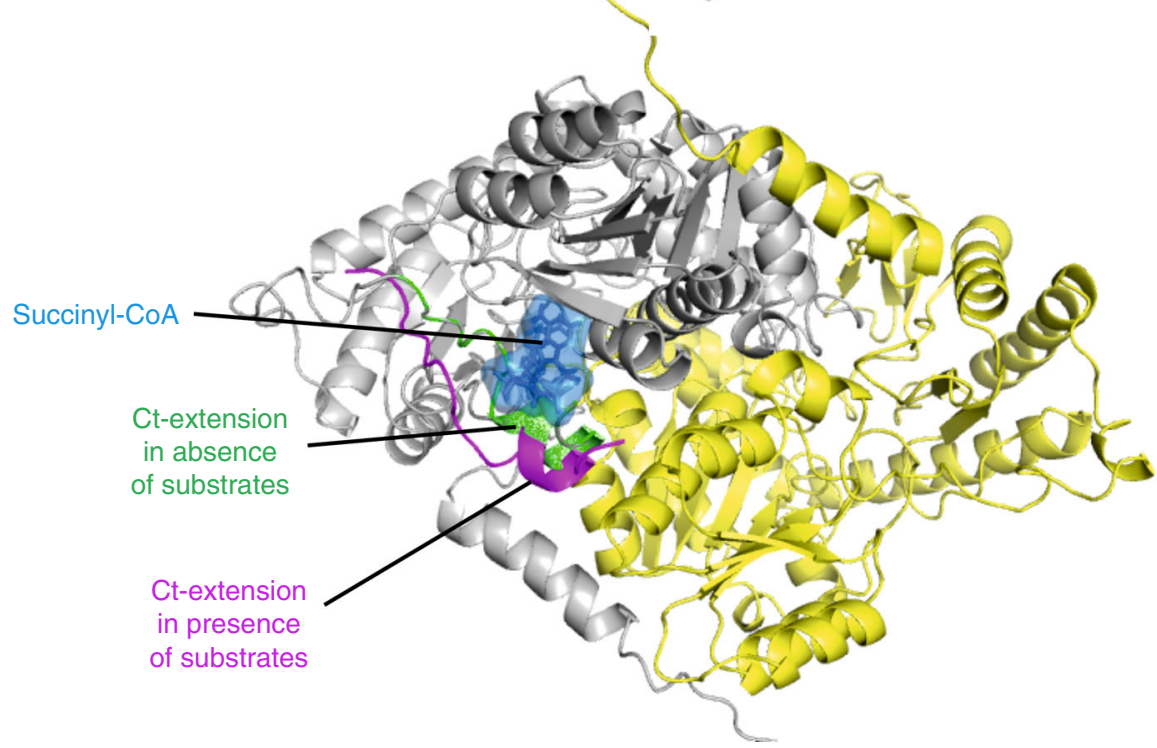

Fig. 4 Dynamics of the Ct-extension revealed by MD simulations. a Salt bridges in the absence (left) and presence (right) of substrates. The structure depicted on the left is the crystal structure subjected to energy minimization, and the structure depicted on the right is a representative snapshot taken from the simulation performed in the presence of substrates. Salt bridges are indicated by dashed lines. Water molecules stabililizing the E569 residue are shown in ball and stick representation. $\mathbf{b}$ The orientation of the Ct-extension in the absence and presence of substrates (shown in green and magenta, respectively). The space occupied by succinyl-CoA in the simulated structure is shown in blue.

leads to an increased anti-correlation between subdomain 2 (containing active site residues) of one subunit and subdomain 3 (containing the Ct-extension) of the other subunit (Supplementary Fig. 13a). The anti-correlation across the two subunits is facilitated by interaction of PLP from one subunit with Thr420 and Thr421 from the other subunit (Supplementary Fig. 13b). Altogether, the simulations data indicate the mobility of the hsALAS2 Ct-extension between different conformations during catalysis.

Revised understanding of disease-causing mutations. The hsALAS2 crystal structure provides a template to understand the molecular basis of disease-causing mutations for XLSA and XLP. To date, over 82 missense mutations affecting 69 different residues of hsALAS2 have been identified as causing XLSA (Supplementary Table 2 and Supplementary Fig. 14). These mutations are largely concentrated in the catalytic core and many have been previously mapped onto the rcALAS structure ${ }^{12}$, allowing the categorization of their putative molecular defects including disruption of the dimerization interface, direct disruption of catalytic regions, or a global destabilization of the enzymatic fold.

The hsALAS2 Ct-extension sheds insights on some previously characterized mutations in the catalytic core, namely D159N and D159Y. The rcALAS open and closed structures (PDB 2BWN) show that formation and disruption of a salt bridge between Glu17
(Asp159 in hsALAS2) and Arg368 (Arg511 in hsALAS2) is key to transitioning between open and closed states (Fig. 3a). Aforementioned in our hsALAS2 structure, Arg511 forms a salt bridge to Glu569 of the Ct-extension, and to Asp159 in helix a 1 of catalytic core (Fig. 3a). Results from our DSF, activity assays and MD simulations (Fig. 3b-e and Supplementary Fig. 12) altogether demonstrate the importance of the Arg511:Glu569 interaction towards the structural integrity of the Ct-extension. By contrast, disruption of the Asp159:Arg511 interaction, the likely culprit for the disease-causing D159N and D159Y variants, may reduce the ability of ALAS2 to adopt the catalytically active closed conformation, and stabilize the interaction of Arg511 with Glu569.

Gain-of-function XLP mutations all locate to exon 11 encoding the hsALAS2 Ct-extension. Three reported indel mutations (Q548X, delAT, and delAGTG) completely delete the two-turn a15 helical region that blocks the ALAS2 active site. A fourth known indel mutation (delG) leaves the Ct-extension helix intact, though it results in a frameshift and an additional 12-aa sequence downstream of that region ${ }^{24}$ (Supplementary Fig. 2). Not surprisingly, the elongated C-terminus of recombinant delAGTG is subject to proteolysis during protein preparation, while the same region of the delG variant is not (Supplementary Fig. 15a). Disruption of the a15 helix would alleviate the restriction of conformational change imposed by the interaction of the 
Table 3 Steady-state kinetics of recombinant hsALAS2 XLP variants.

\begin{tabular}{|c|c|c|c|c|c|c|c|}
\hline$\overline{\text { WT }}$ & $3.5 \pm 0.2$ & $3600 \pm 200$ & $32 \pm 4$ & $0.110 \pm 0.014$ & $3440 \pm 90$ & $8.5 \pm 0.8$ & $0.41 \pm 0.04$ \\
\hline Q548X & $17.3 \pm 1.1$ & $19,900 \pm 1000$ & $55 \pm 5$ & $0.31 \pm 0.04$ & $17700 \pm 330$ & $12.4 \pm 0.6$ & $1.40 \pm 0.11$ \\
\hline delG & $4.5 \pm 0.2$ & $4000 \pm 180$ & $20 \pm 3$ & $0.23 \pm 0.03$ & $4960 \pm 140$ & $6.7 \pm 0.7$ & $0.66 \pm 0.08$ \\
\hline
\end{tabular}

Kinetic parameters for recombinant His 6 -tagged hsALAS2 ${ }_{\triangle N} 78$ WT and XLP variant proteins expressed in E. coli. Reported parameters are best-fit values \pm SE for progression curves (Supplementary Fig. 15) derived from the Prism software (GraphPad 8.0). Source data are provided as a Source Data file.

Ct-extension with the active site loop, providing an explanation for the increased rates of product release as detailed previously in stopped-flow experiments ${ }^{9}$. Our steady-state kinetic analysis of intact recombinant hsALAS2 WT and XLP variants (Supplementary Fig. $15 \mathrm{~b}, \mathrm{c})$ reveals that the $k_{\text {cat }}$ and $V_{\max }$ values for delAT and Q548X are 3- to 6-fold greater than WT and delG proteins (Table 3), in agreement with those reported in the literature 9,24 (delAGTG was not assayed due to C-terminal proteolysis and the putative formation of heterodimers). The association of the delG mutation with XLP has been hypothesized to be the result of increased in vivo enzyme stability relative to that of $\mathrm{WT}^{24}$. We show here that delG has the same 2- to 3-fold wild-type efficiency $\left(k_{\text {cat }} / K_{\mathrm{m}}\right)$ with respect to succinyl-CoA as delAT and Q548X (Table 3). Interestingly, this appears to be due in part to the lower $K_{\mathrm{m}}$ of delG for succinyl-CoA compared to delAT, Q548X, and WT forms, suggesting that delG has a greater affinity for succinylCoA and this may contribute to its hyperactivity.

Crystallography-based fragment screening. We performed crystallography-based fragment screening to identify chemical ligands that serve as a starting point for development of small molecule therapy towards inherited defects of erythroid heme biosynthesis situated downstream of the ALAS2 reaction step, via a substrate reduction approach. Through solving $295 \mathrm{X}$-ray data sets (1.44-1.93 $\AA$ resolution) from hsALAS $2_{\triangle \mathrm{N} 142}$ crystals, each soaked with a different compound from a diverse fragment library, we identified 9 co-crystal structures each with a fragment bound to a hotspot region surrounding the Ct-extension (Fig. 5a and Supplementary Table 3 and Supplementary Data 1).

Fragments 1-8 (Fig. 5b) are accommodated in a pocket between helix $\alpha 15$ of one subunit and its interacting regions (helix a4) in the opposite subunit (Fig. 5c). This pocket is mainly hydrophobic in nature, contributed by Tyr 574 from helix $\alpha 15$ and residues Phe267, Thr268, Lys271, Ile272, and Tyr413 from the opposite chain. Five of the fragments (1-5) extend into a subsite (referred to as ' $I a$ ' in Fig. 5c) packing against Glu155 and Lys152 from the first helix ( $\alpha 1)$ of the catalytic core. As described above, helix al stabilizes the active site mobile loop and C-terminal helix a15, with contributions from Asp159 that forms the network of charged interactions among Asp159:Arg511:Glu569. The aromatic side-chain of Tyr574 (helix a15) adopts an alternate conformation when bound to fragments 2-4 (Supplementary Fig. 16). Three other fragments (6-8) occupy another subsite (' $I b$ ' in Fig. 5c) in direct proximity to the last turn of helix a15 (e.g. Phe575 and Gly576).

A ninth fragment (9, Fig. 5b) was found at the opposite side of C-terminal helix $\alpha 15$ with respect to the above 8 fragments (Fig. 5c). This site is situated along the 2 -fold axis of the hsALAS2 homodimer. One side of the fragment contacts a hydrophobic surface (Asn294, Ser295, Gly296, and Lys271) from its own subunit; its nitrile group captures hydrogen bonds with the main chain carbonyl of Phe575 (in C-terminal helix a15) and guanidinium side-chain from Arg293. The opposite face of the fragment forms van der Waals contact with the same fragment bound to the dimeric subunit $\left(\mathbf{9}^{\prime}\right)$.

We next interrogated the effects of the nine fragments in solution using our colorimetric activity assay. Activity of hsALAS 2 was moderately inhibited by nearly $15 \%$ in the presence of $1 \mathrm{mM}$ fragments 1 or 8 (Supplementary Fig. 17a), an effect that was not due to fragments alone interfering with the assay signal, and was also dependent on fragment concentration, reaching 28 and $22 \%$ inhibition with $5 \mathrm{mM}$ fragments 1 and 8 , respectively (Supplementary Table 4). MD simulations of holo hsALAS2 in the presence of fragment 1 further showed that the fragment remained bound to one protomer of the homodimer throughout the $50 \mathrm{~ns}$ interval and led to enhanced stabilization of R293-E571 salt bridge (Supplementary Fig. 17b), compared to fragment-free hsALAS2. Our data therefore suggest that hsALAS2 catalytic activity can be potentially modulated by small molecules that target the region close to the Ct-extension.

\section{Discussion}

ALAS2 represents one of a handful of metabolic enzymes, where different mutations within the same protein can lead to distinct disorders associated with loss-of-function (enzyme deficiency) or gain-of-function (enzyme superactivity) disorders ${ }^{40}$. In particular, this study uncovers an autoinhibitory mechanism of ALAS2 mediated by its Ct-extension, providing the framework to explain how frameshift indel mutations in the ALAS2 C-terminus lead to the gain-of-function disorder XLP.

Previously, a conserved mobile loop in the rcALAS active site, undergoing conformational change to bind succinyl-CoA, was proposed as a regulatory feature that commited the enzyme to catalysis through an open-to-close active site transition ${ }^{12}$. Here, the hsALAS2 Ct-extension imposes additional conformational restriction on this active site loop (through the salt bridge network Glu569:Arg511:Asp159), preventing transition to the open conformation. To overcome these restraints, displacement of the Ctextension from the conformation depicted in our structure, as supported by our MD simulations, is hence essential for both substrate binding and product release. Our data therefore add to the described complexity of regulatory mechanisms known to exist to modulate ALAS2 activity 41 , underlining the importance of its tight regulation to maintain metabolic flux during erythropoiesis.

It is of note that others have shown that the Ct-extension of hsALAS2 most significantly impacts ALA release after succinylCoA binding9, suggesting why product release is the ratedetermining step. Previous studies ${ }^{24}$ have highlighted that truncation of the Ct-extension does not significantly affect the $K_{\mathrm{m}}$ for either substrate. Our data here, however, indicate that a lower $K_{\mathrm{m}}$ for the delG XLP variant may contribute to its elevated catalytic efficiency. Transient kinetics monitoring the formation and decay of the intermediate quinoid species pointed to an increase in the rate of product release in the truncated forms of ALAS2 (ref. ${ }^{9}$ ). Our structure also provides insight into the role of engineered mutations of the active site loop from previous studies ${ }^{33}$ 
a

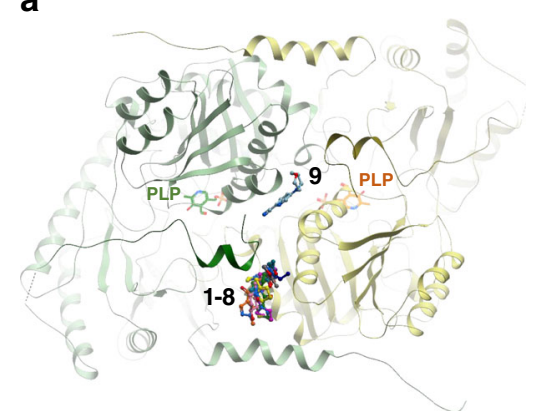

b
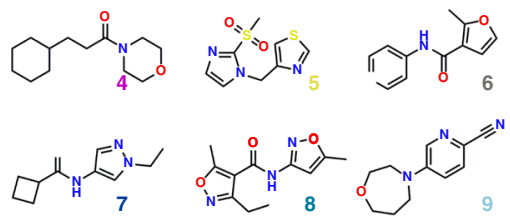

C

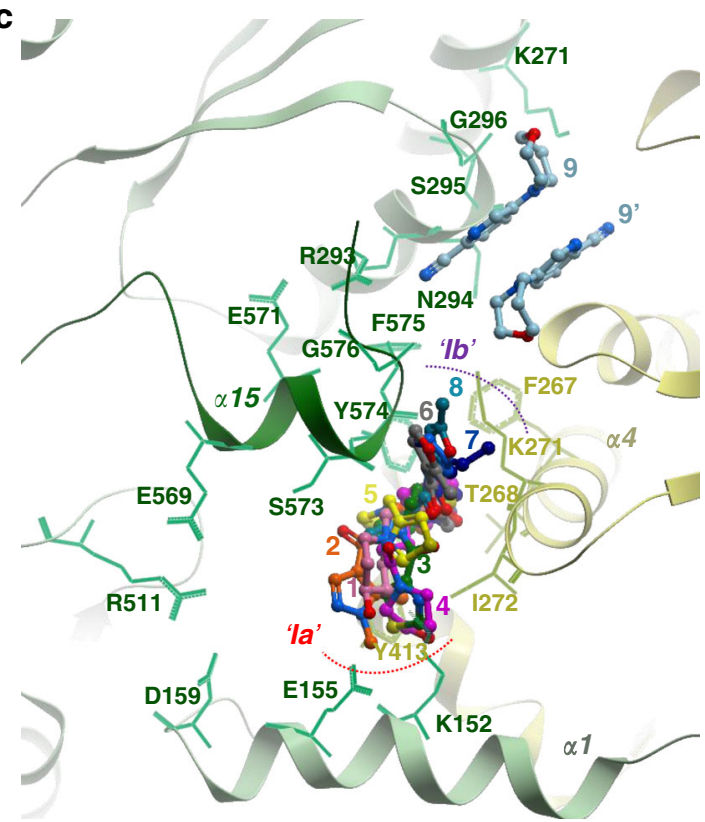

Fig. 5 Crystallography-based fragment screening of hsALAS2. a ALAS2-bound fragments are clustered into distinct regions of the protein. The two subunits of an ALAS2 homodimer are colored green and yellow. b Chemical structures of the fragments 1-9, which are bound in proximity to the hsALAS2 Ct-extension. c Close-up view of the Ct-extension region of one subunit (green) and its neighboring subunit (yellow), showing the binding modes of fragments 1-9. For fragment $\mathbf{9}$, the second molecule $\left(\mathbf{9}^{\prime}\right)$ bound to the yellow dimeric subunit is also shown.

Specifically, engineered mutations directed to the active site loop of murine ALAS2 also lead to a similar hyperactivity as the clinical mutations causing XLP. This observation can be easily reconciled with the hsALAS2 structure, as it would likely abolish interactions with the Ct-extension helix $\alpha 15$ and relieve the autoinhibition.

We speculate that the C-terminal regulatory feature, observed here for ALAS2, will also be present with a similar role in the more ubiquitous ALAS1 isozyme, considering their sequence conservation within this region (e.g. helix a15). However, beyond helix $\alpha 15$ at the far C-terminus, ALAS1 and ALAS2 have evolved very diverse amino acid sequences. Being surface-exposed, these residues may endow the two isoforms with different protein interaction functions. For example, the hsALAS2 Ct-extension is implicated from in vitro pull-down studies ${ }^{24,36,37}$, as the binding site for the beta subunit SUCLA2 of the succinyl-CoA synthetase (SCS) complex in the TCA cycle ${ }^{42}$. It has been suggested that during erythropoiesis, the ALAS2-SUCLA2 interaction may play a role in directing the cellular pool of succinyl-CoA towards heme biosynthesis, away from its conversion to succinate in the TCA cycle ${ }^{43}$. Additionally, ALAS2 has been shown to be part of a larger heme synthesis metabolon whose role is currently undefined, but may involve coordinated regulation of ALAS2 and FECH, the last enzyme in the multi-step heme biosynthesis pathway ${ }^{44}$. Future studies may determine if and how these protein-protein interactions mediate the ALAS2 Ct-extension to regulate catalysis, most likely through the autoinhibitory mechanism shown in this study, but potentially also through facilitating the PLP loading process of the enzyme, shown to involve the AAA + protease $\mathrm{ClpX}^{26}$.

Finally, our structural data provide a starting point for small molecule drug discovery. Three porphyria disorders can be classified as erythroid-specific, associated with loss of enzyme function in the heme biosynthetic pathway downstream of the ALAS2 step (Supplementary Fig. 18). These porphyrias result in the accumulation of toxic heme intermediates and point to the inhibition of ALAS2 as a potential therapeutic target via the substrate reduction approach that has precedence in other inherited metabolic disorders ${ }^{45}$. This is supported by the recent siRNA knockdown of the ALAS1 mRNA showing effective prevention of adverse effects from acute hepatic porphyrias ${ }^{46,47}$, pointing to a similar therapeutic benefit of ALAS2 inhibition for porphyrias in the erythropoietic pathway. Small molecule inhibitors that target the ALAS2 active site such as the itaconic acid derivative itaconyl-CoA ${ }^{48}$, or take advantage of the enzyme's autoinhibitory mechanism mediated by the Ct-extension, are potential avenues for drug discovery. Relevant to this, fragments identified from our crystallography-based screening campaign have the potential for chemistry optimization into lead inhibitors in the future, aimed at increasing residence time at the Ct-extension region to prevent substrate access and product release during catalysis.

\section{Methods}

Protein expression and purification. DNA fragments encoding hsALAS2 harboring different $\mathrm{N}$ - and C-terminal boundaries (Supplementary Fig. 2) were cloned into the pFB-LIC-Bse vector in-frame with a tobacco etch virus protease cleavable $\mathrm{N}$-terminal $\mathrm{His}_{6}$-tag and expressed in baculovirus-infected Sf9 insect cells (Invitrogen) in Sf9 media. Site-directed mutagenesis (R511E) was carried out on hsA$\mathrm{LAS}_{\triangle \mathrm{N} 142}$ and hsALAS2 $2_{\triangle \mathrm{N} 142 \Delta \mathrm{C} 545}$ cloned into pNIC28-Bsa4 vector using the QuikChange method, and confirmed by sequencing. In addition, hsALAS2 ${ }_{\triangle \mathrm{N} 78}$ containing NheI and BamHI restriction sites was cloned into the pET28a vector inframe with the N-terminal $\mathrm{His}_{6}$-tag. Variants of the hsALAS2 ${ }_{\triangle \mathrm{N} 78}$ :pET28a plasmid were prepared via Quickchange mutagenesis, including R511E, E569Q, and XLP mutants delAT, delAGTG, delG, and Q548X. All pET28a fusion proteins were transformed into BL21(DE3) competent cells and single-colony inoculations were induced at an $\mathrm{OD}_{600}$ of $0.5-0.7$ with $0.1 \mathrm{mM}$ IPTG for $16-18 \mathrm{~h}$ at $12{ }^{\circ} \mathrm{C}$. Cultures were then centrifuged and cell pellets stored at $-80^{\circ} \mathrm{C}$.

Proteins expressed in pFB-LIC-Bse and pNIC28-Bsa4 were lysed by sonication and centrifuged at $35,000 \times g$. The clarified cell extract was incubated with $2.5 \mathrm{~mL}$ of Ni-NTA resin pre-equilibrated with lysis buffer $(50 \mathrm{mM}$ HEPES pH 7.5, $500 \mathrm{mM}$ $\mathrm{NaCl}, 10 \mathrm{mM}$ imidazole, $5 \%$ Glycerol, $0.5 \mathrm{mM}$ TCEP). The column was washed with $100 \mathrm{~mL}$ Binding Buffer (50 mM HEPES pH 7.5, $500 \mathrm{mM} \mathrm{NaCl}, 5 \%$ glycerol, $10 \mathrm{mM}$ imidazole, $0.5 \mathrm{mM}$ TCEP), $50 \mathrm{~mL}$ wash buffer (50 mM HEPES pH 7.5, $500 \mathrm{mM} \mathrm{NaCl}, 5 \%$ glycerol, $40 \mathrm{mM}$ imidazole, $0.5 \mathrm{mM}$ TCEP) and eluted with $15 \mathrm{~mL}$ of Elution Buffer (50 mM HEPES pH 7.5, $500 \mathrm{mM} \mathrm{NaCl}, 5 \%$ glycerol, $250 \mathrm{mM}$ imidazole, $0.5 \mathrm{mM}$ TCEP). The eluant fractions were concentrated to $5 \mathrm{~mL}$ and applied to a Superdex $20016 / 60$ column pre-equilibrated in GF Buffer (50 mM HEPES pH 7.5, $500 \mathrm{mM} \mathrm{NaCl}, 0.5 \mathrm{mM}$ TCEP, $5 \%$ glycerol). Eluted protein fractions were pooled and concentrated to $10-17 \mathrm{mg} \mathrm{mL}^{-1}$. Where applicable, $\mathrm{His}_{6}$-tag removal was carried out by overnight treatment with TEV protease, followed by purification over Ni-NTA resin. 
The hsALAS2 variants expressed in pET28a were similarly purified. Specifically, each protein was captured on a HisPur Cobalt Resin (Thermo Scientific) in solubilization buffer consisting of $50 \mathrm{mM}$ Tris- $\mathrm{HCl}, \mathrm{pH}$ 8.0, $20 \mu \mathrm{M}$ PLP, $100 \mathrm{mM}$ $\mathrm{KCl}, 10 \%$ glycerol, and $0.2 \%$ Tween-20. The resin was washed with solubilization buffer containing $15 \mathrm{mM}$ imidazole. PMSF was also added at $0.5 \mathrm{mM}$ during sonication, binding and washing steps. Elutions in $250 \mathrm{mM}$ imidazole were buffer exchanged over Sephadex G-25 spin columns and quantitated with the Pierce BCA Protein Assay Kit (Thermo Scientific). Purified protein samples ( $1 \mu \mathrm{g}$ for SDSPAGE and $100 \mathrm{ng}$ for western blots) were run out on Mini-PROTEAN Stain-Free polyacrylamide gels (Bio-Rad) and bands detected using the ChemiDoc MP imaging system (Bio-Rad) and ImageLab software (version 5.1, Bio-Rad). Multiplex western blotting was carried out by transferring gel bands to low-fluorescence PVDF membrane, followed by blocking the membrane in $2 \%$ BSA at room temperature for one hour and incubation overnight at $4^{\circ} \mathrm{C}$ in 1:10,000 rabbit whole-serum polyclonal hsALAS2 (custom-made, purified antibody from Invitrogen available through Thermo Scientific, catalog number 21553) and 1:5000 mouse monoclonal anti-6xHis (Proteintech, catalog number 66005-1-lg) primary antibodies in 1\% BSA. After washing extensively in TBST, the membrane was incubated in 1:50,000 dilutions of Invitrogen fluorescent donkey anti-rabbit IgG (DyLight 550, catalog number SA5-10039) and goat anti-mouse IgG (DyLight 650, catalog number SA5-10041) secondary antibodies (Thermo Scientific) before washing again and imaging on the ChemiDoc system. Uncropped and unprocessed scans of blots and gels are provided in the Source Data file.

Crystallization and structure determination. Crystals were grown by the vapor diffusion method. To crystallize hsALAS2 ${ }_{\triangle N 142}$, protein was pre-incubated with $5 \mathrm{mM}$ hydroxylamine for $30 \mathrm{~min}$ on ice to convert PLP into the non-covalent form. Sitting drops containing $75 \mathrm{~nL}$ protein $\left(17 \mathrm{mg} \mathrm{mL}^{-1}\right)$ and $75 \mathrm{~nL}$ well solution containing 25\% (w/v) PEG 3350, $0.1 \mathrm{M}$ Bis-Tris $\mathrm{pH} 6.7$ and $0.3 \mathrm{M} \mathrm{MgCl}_{2}$ were equilibrated at $20^{\circ} \mathrm{C}$. To crystallize hsALAS2 ${ }_{\triangle \mathrm{N} 78}$, sitting drops containing $200 \mathrm{~nL}$ protein $\left(11 \mathrm{mg} \mathrm{mL}^{-1}\right), 133 \mathrm{~nL}$ well solution containing $20 \%$ (w/v) PEG3350 and $0.2 \mathrm{M} \mathrm{NH}_{4} \mathrm{Cl}$, and $67 \mathrm{~nL}$ seed solution derived from previously obtained micro crystals. Crystals were cryo-protected using $25 \%$ (v/v) ethylene glycol and flashcooled in liquid nitrogen. Diffraction data were collected at the DLS beamline I04 and I24.

HsALAS2 ${ }_{\triangle \mathrm{N} 142}$ was crystallized in the monoclinic space group C2 with two molecules in the asymmetric unit. The data was processed using the Xia2 autoprocessing dials pipeline.

hsALAS2 ${ }_{\triangle \mathrm{N} 78}$ was crystallized in the monoclinic space group $\mathrm{P} 2_{1}$ with four molecules in the asymmetric unit. The data were processed using non-ellipsoidal anisotropy truncations by the STARANISO server, based on a lower limit of 1.2 for the local I/sigI producing resolution cutoff of $2.65 \AA$ in the best direction and $3.55 \AA$ in worst. The human structures were solved by molecular replacement using the program PHASER from CCP4 (ref. ${ }^{49}$ ) and the rcALAS structure (PDB $2 \mathrm{BWN})^{12}$ as the search model. The final model was produced by iterative cycles of restrained refinement and model building using COOT $^{50}$, REFMAC5 from CCP4 (ref. ${ }^{51}$ ) and Phenix.refine ${ }^{52}$

The final model consists of Phe143-Met578 of chains A and B. In both chains the last 9 residues, aa 579-587, and a loop between Ser 182 and Ser188 were not visible in the electron density map indicating that this region was largely unstructured. Additionally, chain-to-chain variation in electron density quality and loop orientation were found between residues Asp549 and Cys558, likely reflective of differing crystal packing restraints and an intrinsic tendency for conformational heterogeneity within these regions. Coordinates and structure factors for hsALAS2 ${ }_{\triangle \mathrm{N} 142}$ were deposited in the PDB with the accession code $6 \mathrm{HRH}$. Statistics for data collection and refinement are summarized in Table 1.

Crystallography-based fragment screening. To grow crystals for the fragment screening campaign, $10 \mathrm{mg} \mathrm{mL}^{-1}$ of hsALAS2 ${ }_{\triangle \mathrm{N} 142}$ was pre-incubated with $5 \mathrm{mM}$ hydroxylamine for $30 \mathrm{~min}$ on ice to convert PLP into the homogenous, noncovalent form. Crystals were grown by vapor diffusion in $400 \mathrm{~nL}$ sitting drops in the presence of seeds at $20^{\circ} \mathrm{C}$ equilibrated against well solutions of $0.1 \mathrm{M}$ Bis-Tris $\mathrm{pH}$ 7.0, 0.3 M magnesium chloride and 23\% PEG3350. For soaking, $50 \mathrm{~nL}$ of each fragment compound (final concentration of $125 \mathrm{mM}$ ) was added to a crystallization drop using an ECHO acoustic liquid handler dispenser at the Diamond light source XChem facility. Crystals were soaked for two hours with fragments from the DSiPoised Library (Diamond Light Source Ltd) before being harvested using XChem SHIFTER technology, cryo-cooled in liquid nitrogen, and data sets collected at the beamline I04-1 in "automated unattended" mode. The XChemXplorer pipeline ${ }^{53}$ was used for structure solution with parallel molecular replacement with DIMPLE from CCP4 (ref. ${ }^{54}$ ), followed by map averaging and statistical modeling to identify weak electron densities generated from low occupancy fragments using PANDDA software ${ }^{55}$. Coordinates and structure factors for data sets with the 9 bound fragments have been deposited in the PDB (Supplementary Table 3), and refinement statistics are found in Supplementary Data 1.

SAXS. SAXS experiments for the hsALAS2 ${ }_{\Delta \mathrm{N} 142}$ and hsALAS2 ${ }_{\triangle \mathrm{N} 142 \Delta \mathrm{C} 545}$ were performed at $0.99 \AA$ wavelength on the Diamond Light Source beamline B21 coupled to the Shodex KW403-4F size exclusion column (Harwell, UK) and equipped with Pilatus $2 \mathrm{M}$ two-dimensional detector at $4.014 \mathrm{~m}$ distance from the sample, $0.005<q<0.4 \AA-1$ ( $q=4 \pi \sin \theta / \lambda, 2 \theta$ is the scattering angle). The samples were prepared in a buffer containing $300 \mathrm{mM} \mathrm{NaCl}, 25 \mathrm{mM}$ Hepes $7.5,1 \mathrm{mM}$ TCEP, $2 \%$ glycerol, $1 \%$ sucrose, and the measurements were performed at $20^{\circ} \mathrm{C}$. The data were processed and analyzed with Scatter ${ }^{56}$ and the ATSAS program package $^{57}$. Scatter was used to calculate the radius of gyration Rg and forward scattering $\mathrm{I}(0)$ via Guinier approximation, and to derive the maximum particle dimension Dmax and $\mathrm{P}(\mathrm{r})$ function. The ab initio model was derived using DAMMIF ${ }^{57}$. Ten individual models were created, then overlaid and averaged using DAMAVER ${ }^{57}$.

Activity assays and kinetics. To measure enzyme activity of hsALAS2 ${ }_{\triangle \mathrm{N} 78}$ and hsALAS2 ${ }_{\triangle \mathrm{N} 142}$ proteins, discontinuous colorimetric activity assays were conducted per Shoolingin-Jordan et al. ${ }^{58}$ with modifications. Reaction mixtures consisted of $50 \mathrm{mM}$ potassium phosphate buffer, $\mathrm{pH} 7.4,50 \mathrm{mM}$ PLP, $1 \mathrm{mM}$ DTT, $10 \mathrm{mM} \mathrm{MgCl}$, various concentrations of glycine and succinyl-CoA (Sigma), and $1-4 \mu \mathrm{g} \mathrm{mL}^{-1}$ freshly purified hsALAS2 enzyme ( $175 \mu \mathrm{L}$ total). After incubation at $37^{\circ} \mathrm{C}$ for $15 \mathrm{~min}$ (previously checked for linear ALA formation with each enzyme concentration), reactions were terminated with $100 \mu \mathrm{L}$ trichloroacetic acid and centrifuged at $13,000 \times g$ for 5 min to remove protein. Supernatants $(240 \mu \mathrm{L})$ were added to freshly prepared mixtures of $240 \mu \mathrm{L}$ of $1 \mathrm{M}$ sodium acetate, $\mathrm{pH} 4.7$, and $20 \mu \mathrm{L}$ acetylacetone ( $500 \mu \mathrm{L}$ total), and boiled for $10 \mathrm{~min}$ to derivatize the ALA product. Samples were cooled and three $150 \mu \mathrm{L}$ aliquots per reaction (two or three technical replicates) were further derivatized with $150 \mu \mathrm{L}$ modified Ehrlich's reagent and monitored at $554 \mathrm{~nm}$ every $60 \mathrm{~s}$ in a CLARIOstar microplate reader (BMG Labtech). Assays containing small-molecule fragments were scaled down to $140 \mu \mathrm{L}$ reaction mixtures (two technical replicates) and included $5 \% \mathrm{v} / \mathrm{v}(7 \mu \mathrm{L})$ of fragment stock solutions or DMSO diluent. Absorbance values collected after $5 \mathrm{~min}$ were converted to molar quantities of ALA using an extinction coefficient of $60.4 \mathrm{mM}^{-1} \mathrm{~cm}^{-1}$. For kinetic studies, apparent $V_{\max }, K_{\mathrm{m}}$, and $k_{\text {cat }}$ values were determined by titrating $2.5-50 \mathrm{mM}$ glycine in the presence of $100 \mu \mathrm{M}$ succinyl-CoA and $5-100 \mu \mathrm{M}$ succinyl-CoA in the presence of $50 \mathrm{mM}$ glycine. Michaelis-Menten nonlinear regression analysis was subsequently carried out on data for two or three separate protein preparations (two or three biological replicates) with Prism software (GraphPad 8.0). As control, we confirmed that the $\mathrm{His}_{6}$-tag on the recombinant protein did not influence hsALAS2 activity, as shown for WT and R511E variant of hsALAS2 $_{\triangle \mathrm{N} 142}$ (Fig. 3d, e and Table 2).

Differential scanning fluorimetry. HsALAS2 ${ }_{\triangle \mathrm{N} 142}$ was assayed for shifts in melting temperature caused by titration of PLP and succinyl-CoA in a 96-well PCR plate using an Mx3005p RT-PCR machine (Stratagene). Each reaction well $(20 \mu \mathrm{l})$ consisted of protein $(20 \mu \mathrm{M}$ in a buffer containing $50 \mathrm{mM}$ Hepes, $\mathrm{pH} 7.5,500 \mathrm{mM}$ $\mathrm{NaCl}$ ), SYPRO-Orange (Invitrogen) diluted 1000×. Fluorescence intensities were measured from $25^{\circ} \mathrm{C}$ to $96^{\circ} \mathrm{C}$ with a ramp rate of $1^{\circ} \mathrm{C} \mathrm{min}-1$, as described ${ }^{59}$. The temperature shifts, $\Delta T_{\mathrm{m}, \mathrm{obs}}$, for each ligand were determined. The experiment was repeated three times and an error was calculated based on the standard deviation.

Flourescence intensity. HsALAS2 ${ }_{\triangle \mathrm{N} 142}$ was assayed for shifts in fluorescence intensity caused by titration of PLP. Each well $(10 \mu \mathrm{l})$ consisted of protein $(20 \mu \mathrm{M}$ in a buffer containing $50 \mathrm{mM}$ Hepes, $\mathrm{pH} 7.5,500 \mathrm{mM} \mathrm{NaCl}$ ) and varying concentrations of PLP. Schiff base formation was monitored via excitation at $440 \mathrm{~nm}$ and emission at $520 \mathrm{~nm}$ using an Omega plate reader. The experiment was repeated three times and an error was calculated based on the standard deviation.

Modeling hsALAS2 for MD simulation. The crystal structure of hsALAS2 (this study) was used for all MD simulation studies. Missing residues were modeled into the crystal structure based on a homology model of hsALAS2 constructed with the scALAS crystal structure (PDB 5TXT) as a template using the MODELLER pro$\operatorname{gram}^{60}$. The homology model was first structurally aligned with the hsALAS2 crystal structure, and the coordinates of the missing residues were then taken from the homology model and incorporated into the crystal structure. The structure generated, after an energy minimization step, thereby included residues 137-578 from both monomeric units. Three different systems were modeled: (i) hsALAS2 apo form (without PLP), (ii) hsALAS2 holo form with PLP covalently attached to Lys391 (but without substrates), and (iii) hsALAS2 holo form with substrates glycine-PLP and succinyl-CoA.

In order to model system (iii), the starting structure for the protein was chosen from the simulation on system (ii) by clustering all conformations sampled in the trajectory and then choosing the structure at the center of the largest cluster as the representative structure. PLP was then removed from Lys391 in the representative structure. The substrates glycine-PLP and succinyl-CoA were modeled into this structure by using the substrate-bound Rhodobacter capsulatus ALAS (rcALAS) structure as a reference template. The structure with PDB ID 2BWP (rcALAS with glycine-PLP) was first aligned with the representative hsALAS2 structure, and the resulting orientation of glycine-PLP was modeled into the representative hsALAS2 structure. In a similar manner, the structure with PDB ID 2BWO (rcALAS with succinyl-CoA) was used for modeling succinyl-CoA into the representative hsALAS2 structure. Both the monomeric units of the representative structure included the two substrates. In order to remove possible bad contacts 
between the substrates and the protein, minimization and equilibration of the system was performed in the presence of restraints on the heavy atoms of the protein (a restraining force with force constant $1000 \mathrm{~kJ} \mathrm{~mol}^{-1} \mathrm{~nm}^{-2}$ was used).

All MD simulations were performed in the NPT ensemble (constant number of particles $(\mathrm{N})$, constant pressure $(\mathrm{P})$, and constant temperature $(\mathrm{T})$ ) for $50 \mathrm{~ns}$ using the GROMACS program with the CHARMM36 all-atom force field ${ }^{61,62}$ and the TIP3P water model ${ }^{63}$ (Supplementary Software 1). A time-step of $2 \mathrm{fs}$ was used, and all covalent bonds involving hydrogen atoms were constrained using the LINCS algorithm ${ }^{64}$. A cut-off of $12 \AA$ was used for short-range non-bonded interactions, and the particle mesh Ewald method was employed for long-range electrostatics ${ }^{65}$. Force field parameters for PLP-lysine, PLP-glycine, and succinylCoA were obtained from the Paramchem server 66,67 . Details of the simulation box for all systems are shown in Supplementary Table 5. Two sets of simulations were performed for all the systems.

To study the conformational dynamics of ALAS2 R511E variant, the crystal structure of hsALAS2 ${ }_{\triangle \mathrm{N} 142}$ was used to perform the R511E substitution using the CHARMM-GUI server ${ }^{68}$. The simulation protocol was the same as that followed for wild-type ALAS2.

Reporting summary. Further information on research design is available in the Nature Research Reporting Summary linked to this article.

\section{Data availability}

All coordinates and structure factors were deposited to the Protein Data Bank under the accession codes PDB 6HRH [https://doi.org/10.2210/pdb6HRH/pdb], PDB 5QQY [https://doi.org/10.2210/pdb5QQY/pdb], PDB 5QR1 [https://doi.org/10.2210/pdb5QR1/ pdb], PDB 5QRA [https://doi.org/10.2210/pdb5QRA/pdb], PDB 5QRC [https://doi.org/ 10.2210/pdb5QRC/pdb], PDB 5QRD [https://doi.org/10.2210/pdb5QRD/pdb], PDB 5QQW [https://doi.org/10.2210/pdb5QQW/pdb], PDB 5QQX [https://doi.org/10.2210/ pdb5QQX/pdb], PDB 5QRE [https://doi.org/10.2210/pdb5QRE/pdb], PDB 5QQU [https://doi.org/10.2210/pdb5QQU/pdb]. Main data supporting the findings of this study are available within the article and the Supplementary Information. Raw data for Tables 2 and 3, Supplementary Table 4, Fig. 3b-e, and Supplementary Figs. 3a-c, 4a-c, 5, 6, 9-12, 13a, 15a-c, and 17a, b are available in the Source Data file. Other data are available from the corresponding author upon reasonable request.

\section{Code availability}

GROMACS codes for MD simulations reported in Supplementary Figs. 9-11, 12b, 13a, and $17 \mathrm{~b}$ are provided as Supplementary Software 1.

Received: 6 July 2019; Accepted: 11 May 2020;

Published online: 04 June 2020

\section{References}

1. Gibson, K. D., Laver, W. G. \& Neuberger, A. Initial stages in the biosynthesis of porphyrins. 2. The formation of delta-aminolaevulic acid from glycine and succinyl-coenzyme A by particles from chicken erythrocytes. Biochem. J. 70, 71-81 (1958).

2. Akhtar, M., Abboud, M. M., Barnard, G., Jordan, P. \& Zaman, Z. Mechanism and stereochemistry of enzymic reactions involved in porphyrin biosynthesis. Philos. Trans. R. Soc. Lond. B Biol. Sci. 273, 117-136 (1976).

3. Dailey, H. A. \& Meissner, P. N. Erythroid heme biosynthesis and its disorders. Cold Spring Harb. Perspect. Med. 3, a011676 (2013).

4. Dailey H. A., Dailey T. A. in The Porphyrin Handbook (eds Kadish, K. M., Smith, K. M. \& Guilard, R.) (Academic Press, 2003).

5. Bishop, D. F., Henderson, A. S. \& Astrin, K. H. Human delta-aminolevulinate synthase: assignment of the housekeeping gene to $3 p 21$ and the erythroidspecific gene to the X chromosome. Genomics 7, 207-214 (1990).

6. Riddle, R. D., Yamamoto, M. \& Engel, J. D. Expression of delta-aminolevulinate synthase in avian cells: separate genes encode erythroid-specific and nonspecific isozymes. Proc. Natl Acad. Sci. USA 86, 792-796 (1989).

7. Besur, S., Hou, W., Schmeltzer, P. \& Bonkovsky, H. L. Clinically important features of porphyrin and heme metabolism and the porphyrias. Metabolites 4 , 977-1006 (2014)

8. Hunter, G. A., Zhang, J. \& Ferreira, G. C. Transient kinetic studies support refinements to the chemical and kinetic mechanisms of aminolevulinate synthase. J. Biol. Chem. 282, 23025-23035 (2007).

9. Fratz, E. J. et al. Human erythroid 5-aminolevulinate synthase mutations associated with X-linked protoporphyria disrupt the conformational equilibrium and enhance product release. Biochemistry 54, 5617-5631 (2015).

10. Stojanovski, B. M., Hunter, G. A., Jahn, M., Jahn, D. \& Ferreira, G. C. Unstable reaction intermediates and hysteresis during the catalytic cycle of 5aminolevulinate synthase: implications from using pseudo and alternate substrates and a promiscuous enzyme variant. J. Biol. Chem. 289, 22915-22925 (2014).

11. Hunter, G. A. \& Ferreira, G. C. Pre-steady-state reaction of 5-aminolevulinate synthase. Evidence for a rate-determining product release. J. Biol. Chem. $\mathbf{2 7 4}$ 12222-12228 (1999)

12. Astner, I. et al. Crystal structure of 5 -aminolevulinate synthase, the first enzyme of heme biosynthesis, and its link to XLSA in humans. EMBO J. $\mathbf{2 4}$, 3166-3177 (2005).

13. Lendrihas, T., Hunter, G. A. \& Ferreira, G. C. Targeting the active site gate to yield hyperactive variants of 5 -aminolevulinate synthase. J. Biol. Chem. 285, 13704-13711 (2010)

14. Munakata, H., Yamagami, T., Nagai, T., Yamamoto, M. \& Hayashi, N. Purification and structure of rat erythroid-specific delta-aminolevulinate synthase. J. Biochem. 114, 103-111 (1993).

15. Srivastava, G. et al. Regulation of 5-aminolevulinate synthase mRNA in different rat tissues. J. Biol. Chem. 263, 5202-5209 (1988).

16. Schoenhaut, D. S. \& Curtis, P. J. Nucleotide sequence of mouse 5 aminolevulinic acid synthase cDNA and expression of its gene in hepatic and erythroid tissues. Gene 48, 55-63 (1986).

17. Cox, T. C. et al. The major splice variant of human 5 -aminolevulinate synthase-2 contributes significantly to erythroid heme biosynthesis. Int. J. Biochem. Cell Biol. 36, 281-295 (2004).

18. Dailey, T. A., Woodruff, J. H. \& Dailey, H. A. Examination of mitochondrial protein targeting of haem synthetic enzymes: in vivo identification of three functional haem-responsive motifs in 5-aminolaevulinate synthase. Biochem. J. 386, 381-386 (2005).

19. Lathrop, J. T. \& Timko, M. P. Regulation by heme of mitochondrial protein transport through a conserved amino acid motif. Science 259, 522-525 (1993).

20. Munakata, $\mathrm{H}$. et al. Role of the heme regulatory motif in the heme-mediated inhibition of mitochondrial import of 5-aminolevulinate synthase. J. Biochem. 136, 233-238 (2004).

21. Whatley, S. D. et al. C-terminal deletions in the ALAS2 gene lead to gain of function and cause X-linked dominant protoporphyria without anemia or iron overload. Am. J. Hum. Genet. 83, 408-414 (2008).

22. Ducamp, S. et al. Molecular and functional analysis of the C-terminal region of human erythroid-specific 5-aminolevulinic synthase associated with Xlinked dominant protoporphyria (XLDPP). Hum. Mol. Genet. 22, 1280-1288 (2013).

23. Balwani, M. et al. Loss-of-function ferrochelatase and gain-of-function erythroid-specific 5-aminolevulinate synthase mutations causing erythropoietic protoporphyria and X-linked protoporphyria in North American patients reveal novel mutations and a high prevalence of X-linked protoporphyria. Mol. Med. 19, 26-35 (2013).

24. Bishop, D. F., Tchaikovskii, V., Nazarenko, I. \& Desnick, R. J. Molecular expression and characterization of erythroid-specific 5-aminolevulinate synthase gain-of-function mutations causing X-linked protoporphyria. Mol. Med. 19, 18-25 (2013).

25. Tchaikovskii, V., Desnick, R. J. \& Bishop, D. F. Molecular expression, characterization and mechanism of ALAS2 gain-of-function mutants. Mol. Med. 25, 4 (2019).

26. Kardon, J. R. et al. Mitochondrial ClpX activates a key enzyme for heme biosynthesis and erythropoiesis. Cell 161, 858-867 (2015).

27. Yien, Y. Y. et al. Mutation in human CLPX elevates levels of deltaaminolevulinate synthase and protoporphyrin IX to promote erythropoietic protoporphyria. Proc. Natl Acad. Sci. USA 114, E8045-E8052 (2017).

28. Harigae, H. \& Furuyama, K. Hereditary sideroblastic anemia: pathophysiology and gene mutations. Int J. Hematol. 92, 425-431 (2010).

29. Ducamp, S. et al. Sideroblastic anemia: molecular analysis of the ALAS2 gene in a series of 29 probands and functional studies of 10 missense mutations. Hum. Mutat. 32, 590-597 (2011).

30. Bottomley, S. S., May, B. K., Cox, T. C., Cotter, P. D. \& Bishop, D. F. Molecular defects of erythroid 5-aminolevulinate synthase in X-linked sideroblastic anemia. J. Bioenerg. Biomembr. 27, 161-168 (1995).

31. Cotter, P. D., Baumann, M. \& Bishop, D. F. Enzymatic defect in "X-linked" sideroblastic anemia: molecular evidence for erythroid delta-aminolevulinate synthase deficiency. Proc. Natl Acad. Sci. USA 89, 4028-4032 (1992).

32. Stenson, P. D. et al. The Human Gene Mutation Database: towards a comprehensive repository of inherited mutation data for medical research, genetic diagnosis and next-generation sequencing studies. Hum. Genet. 136, 665-677 (2017)

33. Kadirvel, S. et al. The carboxyl-terminal region of erythroid-specific 5aminolevulinate synthase acts as an intrinsic modifier for its catalytic activity and protein stability. Exp. Hematol. 40, 477-486.el (2012).

34. Brown, B. L., Kardon, J. R., Sauer, R. T. \& Baker, T. A. Structure of the mitochondrial aminolevulinic acid synthase, a key heme biosynthetic enzyme. Structure 26, 580-589.e4 (2018) 
35. Yadav, P. K., Xie, P. \& Banerjee, R. Allosteric communication between the pyridoxal $5^{\prime}$-phosphate (PLP) and heme sites in the H2S generator human cystathionine beta-synthase. J. Biol. Chem. 287, 37611-37620 (2012).

36. Bishop, D. F., Tchaikovskii, V., Hoffbrand, A. V., Fraser, M. E. \& Margolis, S. $\mathrm{X}$-linked sideroblastic anemia due to carboxyl-terminal ALAS2 mutations that cause loss of binding to the beta-subunit of succinyl-CoA synthetase (SUCLA2). J. Biol. Chem. 287, 28943-28955 (2012).

37. Furuyama, K. \& Sassa, S. Interaction between succinyl CoA synthetase and the heme-biosynthetic enzyme ALAS-E is disrupted in sideroblastic anemia. J. Clin. Invest. 105, 757-764 (2000).

38. Na I., Catena D., Kong M. J., Ferreira G. C., Uversky V. N. Anti-correlation between the dynamics of the active site loop and C-terminal tail in relation to the homodimer asymmetry of the mouse erythroid 5-aminolevulinate synthase. Int. J. Mol. Sci. 19, 1899 (2018)

39. Swaminathan, S., Harte, W. E. \& Beveridge, D. L. Investigation of domain structure in proteins via molecular dynamics simulation: application to HIV-1 protease dimer. J. Am. Chem. Soc. 113, 2717-2721 (1991).

40. Ferreira C. R., van Karnebeek C. D. M., Vockley J., Blau N. A proposed nosology of inborn errors of metabolism. Genet. Med. 21, 1 (2018).

41. Peoc'h, K. et al. Regulation and tissue-specific expression of deltaaminolevulinic acid synthases in non-syndromic sideroblastic anemias and porphyrias. Mol. Genet. Metab. 128, 190-197 (2019).

42. Lambeth, D. O., Tews, K. N., Adkins, S., Frohlich, D. \& Milavetz, B. I. Expression of two succinyl-CoA synthetases with different nucleotide specificities in mammalian tissues. J. Biol. Chem. 279, 36621-36624 (2004).

43. Burch, J. S. et al. Glutamine via alpha-ketoglutarate dehydrogenase provides succinyl-CoA for heme synthesis during erythropoiesis. Blood 132, 987-998 (2018).

44. Medlock, A. E. et al. Identification of the mitochondrial heme metabolism complex. PLoS ONE 10, e0135896 (2015).

45. Yue W. W., Mackinnon S., Bezerra G. A. Substrate reduction therapy for inborn errors of metabolism. Emerg. Top. Life Sci. 3, 63-73 (2019).

46. Sardh, E. et al. Phase 1 trial of an RNA interference therapy for acute intermittent porphyria. N. Engl. J. Med. 380, 549-558 (2019)

47. Yasuda, M. et al. RNAi-mediated silencing of hepatic Alas1 effectively prevents and treats the induced acute attacks in acute intermittent porphyria mice. Proc. Natl Acad. Sci. USA 111, 7777-7782 (2014).

48. Marcero, J. R. et al. The role of TCA cycle compounds in erythroid heme synthesis and its regulation during an inflammatory response. Blood 130, 3473-3473 (2017).

49. McCoy, A. J., Grosse-Kunstleve, R. W., Storoni, L. C. \& Read, R. J. Likelihoodenhanced fast translation functions. Acta Crystallogr. D Biol. Crystallogr. 61, 458-464 (2005).

50. Emsley, P. \& Cowtan, K. Coot: model-building tools for molecular graphics. Acta Crystallogr. D Biol. Crystallogr. 60, 2126-2132 (2004)

51. Murshudov, G. N., Vagin, A. A. \& Dodson, E. J. Refinement of macromolecular structures by the maximum-likelihood method. Acta Crystallogr. D Biol. Crystallogr. 53, 240-255 (1997)

52. Afonine, P. V. et al. Towards automated crystallographic structure refinement with phenix.refine. Acta Crystallogr. D Biol. Crystallogr. 68, 352-367 (2012).

53. Krojer, T. et al. The XChemExplorer graphical workflow tool for routine or large-scale protein-ligand structure determination. Acta Crystallogr. D Struct. Biol. 73, 267-278 (2017)

54. Winn, M. D. et al. Overview of the CCP4 suite and current developments. Acta Crystallogr. D Biol. Crystallogr. 67, 235-242 (2011).

55. Pearce, N. M. et al. A multi-crystal method for extracting obscured crystallographic states from conventionally uninterpretable electron density. Nat. Commun. 8, 15123 (2017)

56. Förster, S., Apostol, L. \& Bras, W. Scatter: software for the analysis of nanoand mesoscale small-angle scattering. J. Appl. Crystallogr. 43, 639-646 (2010).

57. Petoukhov, M. V. et al. New developments in the ATSAS program package for small-angle scattering data analysis. J. Appl. Crystallogr. 45, 342-350 (2012).

58. Shoolingin-Jordan, P. M., LeLean, J. E. \& Lloyd, A. J. Continuous coupled assay for 5-aminolevulinate synthase. Methods Enzymol. 281, 309-316 (1997).

59. Niesen, F. H., Berglund, H. \& Vedadi, M. The use of differential scanning fluorimetry to detect ligand interactions that promote protein stability. Nat. Protoc. 2, 2212-2221 (2007).

60. Sali, A. \& Blundell, T. L. Comparative protein modelling by satisfaction of spatial restraints. J. Mol. Biol. 234, 779-815 (1993).

61. Best, R. B. et al. Optimization of the additive CHARMM all-atom protein force field targeting improved sampling of the backbone phi, psi and sidechain chi(1) and chi(2) dihedral angles. J. Chem. Theory Comput. 8, 3257-3273 (2012).
62. Abraham, M. J. et al. GROMACS: high performance molecular simulations through multi-level parallelism from laptops to supercomputers. SoftwareX 1 2, 19-25 (2015)

63. Jorgensen, W. L., Chandrasekhar, J., Madura, J. D., Impey, R. W. \& Klein, M. L. Comparison of simple potential functions for simulating liquid water. $J$. Chem. Phys. 79, 926-935 (1983).

64. Hess, B., Bekker, H., Berendsen, H. J. C. \& Fraaije, J. G. E. M. LINCS: a linear constraint solver for molecular simulations. J. Comput. Chem. 18, 1463-1472 (1997).

65. Essmann, U. et al. A smooth particle mesh Ewald method. J. Chem. Phys. 103, 8577-8593 (1995).

66. Vanommeslaeghe, K. \& MacKerell, A. D. Jr. Automation of the CHARMM General Force Field (CGenFF) I: bond perception and atom typing. J. Chem. Inf. Model 52, 3144-3154 (2012).

67. Vanommeslaeghe, K., Raman, E. P. \& MacKerell, A. D. Jr. Automation of the CHARMM General Force Field (CGenFF) II: assignment of bonded parameters and partial atomic charges. J. Chem. Inf. Model 52, 3155-3168 (2012).

68. Jo, S., Kim, T., Iyer, V. G. \& Im, W. CHARMM-GUI: a web-based graphical user interface for CHARMM. J. Comput. Chem. 29, 1859-1865 (2008).

\section{Acknowledgements}

The Structural Genomics Consortium is a registered charity (Number 1097737) that receives funds from AbbVie, Bayer Pharma AG, Boehringer Ingelheim, Canada Foundation for Innovation, Eshelman Institute for Innovation, Genome Canada, Innovative Medicines Initiative (EU/EFPIA) [ULTRA-DD grant no. 115766], Janssen, Merck \& Co., Novartis Pharma AG, Ontario Ministry of Economic Development and Innovation, Pfizer, São Paulo Research Foundation-FAPESP, Takeda, and Wellcome Trust [092809/ Z/10/Z]. H.J.B. is further supported by the Nuffield Department of Clinical Medicine and Medical Research Council. R.J.D. and D.F.B. were supported in part by the Department of Genetics and Genomic Sciences, Icahn School of Medicine at Mount Sinai.

\section{Author contributions}

G.A.B., H.A.D., and W.W.Y. designed the experiments. H.J.B., G.A.B., J.R.M., S.P., W.R.F., E.R., A.R., and D.F.B. performed the experiments. H.J.B., J.R.M., R.J.D., G.B., H.A.D., and W.W.Y. wrote the manuscript.

\section{Competing interests}

The authors declare no competing interests.

\section{Additional information}

Supplementary information is available for this paper at https://doi.org/10.1038/s41467 020-16586-x.

Correspondence and requests for materials should be addressed to W.W.Y.

Peer review information Nature Communications thanks Ruma Banerjee, Chris Oostenbrink and the other, anonymous, reviewer(s) for their contribution to the peer review of this work. Peer reviewer reports are available.

Reprints and permission information is available at http://www.nature.com/reprints

Publisher's note Springer Nature remains neutral with regard to jurisdictional claims in published maps and institutional affiliations.

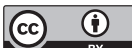

Open Access This article is licensed under a Creative Commons Attribution 4.0 International License, which permits use, sharing, adaptation, distribution and reproduction in any medium or format, as long as you give appropriate credit to the original author(s) and the source, provide a link to the Creative Commons license, and indicate if changes were made. The images or other third party material in this article are included in the article's Creative Commons license, unless indicated otherwise in a credit line to the material. If material is not included in the article's Creative Commons license and your intended use is not permitted by statutory regulation or exceeds the permitted use, you will need to obtain permission directly from the copyright holder. To view a copy of this license, visit http://creativecommons.org/ licenses/by/4.0/.

(c) The Author(s) 2020 